\title{
Dokumentation Soziale Herkunft und wissenschaftliche Vorbildung des Seeoffiziers der Kaiserlichen Marine vor $1914^{1}$
}

In der militärgeschichtlichen Literatur existiert bis heute noch keine eingehende soziologische Untersuchung des kaiserlichen Seeoffizierkorps. Obwohl seit dem Ende des Zweiten Weltkrieges mehrere Arbeiten über die soziale Struktur des Offizierkorps der Armee im Wilhelminischen Zeitalter veröffentlicht wurden 2 , gibt es über das Seeoffizierkorps bisher nur zwei wissenschaftliche Studien ${ }^{3}$. Das ist insofern zu bedauern, als die Kaiserliche Marine als deutsche Teilstreitkraft das Wilhelminische Reich besser repräsentierte als die königlich-preußische Armee 4 . Die Seeoffiziere der Zeit haben sich als *weltoffen * und "weltgewandt* angesehen, ein Urteil, das von Historikern sowie Schriftstellern übernommen wurde. Ein Beispiel für diese Einstellung bieten die Erinnerungen von Graf Zedlitz-Trützschler, Hofmarschall in Berlin, der zu dieser Frage schrieb: "Ihr Gesichtskreis ist ein weiter, ihr Berufsleben fördert die innere Entwicklung; die Vielseitigkeit, der Ernst und die Verantwortlichkeit ihrer Beschäftigung regt Intelligenz, Energie und Selbständigkeit an, erzeugt geistige Interessen und beseitigt Vorurteile ${ }^{5}$." Diese These von der "weltoffenen* Einstellung des Seeoffizierkorps wurde von fast allen Historikern der Kaiserlichen Marine übernommen. Demeter schrieb dem kaiserlichen Seeoffizier eine "gewisse Weltoffenheit « zu ${ }^{B}$. Steinberg charakterisierte die Seeoffiziere als nmen of middle-class backgrounds ", ohne "the caste prejudice, snobbery and aristocratic behaviour of the Army «. Er sah die Marine als einen Triumph der deutschen Liberalen, der $1848 \mathrm{er}$, an: "In a sense, Kultur was an ideology of Germanism ... whether in a battleship or a Wagnerian myth ${ }^{7}$." Für Drascher, der die erste soziologische Untersuchung des kaiserlichen Seeoffizierkorps vorgelegt hat, war der Seeoffizier "ein besonderer soziologischer Typ im Kaiserreich... Weltgewandt, weit gereist $^{\mathrm{B}}$, die militärische Haltung bester preußischer Tradition mit Aufgeschlos-

1 Diese Arbeit wurde durch das Klaus-Epstein-Gedächtnis-Stipendium der Alexander v. Humboldt-Stiftung unterstützt. Die hier angesprochenen Probleme werden in meiner vor dem Abschluß stehenden Dissertation *The German Naval Officer Corps: A Social-Political History, 1890-1918 " eingehender behandelt. Eine Behandlung der anderen Korps der Marine wird dort zu finden sein.

2 S. dazu K. Demeter: Das Deutsche Offizierkorps in Gesellschaft und Staat 1650-1945, Frankfurt, 4. Aufl. 1965 (zit. Demeter); M. Kitchen: The German Officer Corps 1890-1914, Oxford 1968; M. Messerschmidt: Werden und Prägung des preußischen Offizierkorps. Ein Uberblidk, in: Offiziere im Bild von Dokumenten aus drei Jahrzehnten, Stuttgart 1964 (= Beiträge zur Militär- und Kriegsgeschichte, hrsg. vom Militärgeschichtlichen Forschungsamt, Bd 6); H. Rumschöttel: Bildung und Herkunft der bayerischen Offiziere 1866 bis 1914. Zur Geschichte von Mentalität und Ideologie des bayerischen Offizierkorps, in: MGM 2/70, S. 81-131 (zit. Rumschöttel).

- W. Drascher: Zur Soziologie des deutschen Seeoffizierkorps, in: Wehrwissenschaftliche Rundschau 12 (1962), (H. 10), S. 555-569 (zit. Drascher); F. Forstmeier: Probleme der Erziehung und Ausbildung in der Kaiserlichen Marine in Abhängigkeit von geistiger Situation und sozialer Struktur, in: Marine-Rundschau 63 (1966), (H. 4), S. 189-198 (zit. Forstmeier).

- Großadmiral Alfred $\nabla$. Tirpitz behauptete später: Die Marine wurde ein Schmelztiegel des Deutschtums.« A. v. Tirpitz: Erinnerungen, Leipzig 1919, S. 127.

5 Graf R. Zedlitz-Trützschler: Zwölf Jahre am deutschen Kaiserhof, Berlin, Leipzig 1925, S. 122.

A Demeter, S. 26一27. Zitat nach Demeter, Frankfurt, 3. Aufl. 1964, S. 47.

7 J. Steinberg: Yesterday's Deterrent. Tirpitz and the Birth of the German Battle-Fleet, London 1965, S. 38. „Ships were . . . floating manifestations of German Kultur.e

B Vl. Dok. 6, 7, 10. 
senheit und Takt verbindend «" . Drascher interpretierte die Weltanschauung der Seeoffiziere als "national und liberal«, was ihnen des öfteren die Gegnerschaft der preußischen Konservativen eingebracht habe. Besonders typisch für diese Einschätzung des Seeoffizierkorps ist Draschers Behauptung: »Bei der Marine aber war die gesellschaftliche Stellung viel weniger ausschlaggebend als die be rufliche Tüchtigkeit ${ }^{10}$."

Diese Untersuchungen wurden jetzt durch die ausgezeidhete Arbeit von Forstmeier gründlich ergänzt. In dieser ersten wissenschaftlichen Untersudhung des kaiserlichen Seeoffizierkorps kommt Forstmeier zu folgendem Ergebnis: „Das Problem, ob Stand oder Wissen, Charakter oder Bildung bei der Aufnahme ins Offizierkorps der Vorzug zu geben sei, wurde in immer neuen, nie befriedigenden Kompromissen zu lösen gesucht ${ }^{11 . « ~ I n ~ e i n e r ~ v o r ~ k u r z e m ~ e r s c h i e n e n e n ~ A r-~}$ beit behauptet Horn, daß durch die Emennung von Tirpitz zum Staatssekretär des Reichs-Marine-Amtes im Jahre 1897 wthe egalitarian, cosmopolitan, and tolerant attitudes«, die Horn in der preußischen Marine des 19. Jahrhunderts erkennen zu können glaubt, wbegan to vanish». Statt dessen habe die Marine nun wincreasing numbers of officers from the aristocracy and upper middle class « aufgenommen. "Thus the brutality and caste system of the Prussian drill field and barracks life was introduced in a service that had formerly avoided all manifestations of Prussian militarism ${ }^{12}$.*

Die folgende Dokumentation versucht zu klären, inwieweit die hier angeführten Behauptungen und Verallgemeinerungen der Wirklichkeit entsprachen, sie will aber keinesfalls eine umfassende soziologische Untersuchung des kaiserlichen Seeoffizierkorps sein. In ihrem Rahmen kann auch nicht auf die Eigenarten der verschiedenen Korps innerhalb der Marine (Marineingenieure, Sanitäts-, Torpeder-, Reserveoffiziere und so weiter eingegangen werden.

II

Aus welchen sozialen Kreisen rekrutierte die Kaiserliche Marine ihren Seeoffizierersatz? Der Prozentsatz von Seeoffizieren adliger Herkunft war nie hoch, selten über $15 \%$. Dabei ist zu bemerken, daß die meisten adligen Seeoffiziere dem niederen, relativ armen preußischen Adel entstammten 19. Auch in der preußischen Armee hatte sich der Prozentsatz adliger Offiziere schon vor 1914 stark verringert ${ }^{14}$. Im Gegensatz zur Kavallerie oder den Garde-du-Corps Regimentern, die den Seeoffizieren sonst als Vorbild dienten ${ }^{15}$, gab es in der Ma-

- Drascher, S. 567.

10 Drascher, S. 561, 568.

11 Forstmeier, S. 197-198.

12 D. Horn: The German Naval Mutinies of World War I, New Jersey 1969, S. 4, 9. Die letzte Behauptung kann nur, wenn überhaupt, auf die Amtsperiode des Generals Albrecht v. Stosch als Chef der Admiralität (1871-1883) angewandt werden. Vgl. Anm. 13 zu Horns Behauptung betr. der wincreasing numbers of officers from the aristocracy $\%$.

13 S. die Arbeit von G. Sandhofer: Dokumente zum militärischen Werdegang des Großadmirals Dönitz, in: MGM 1/67, S. 61 (zit. Sandhofer). Danach gab es in der Crew von 1907 nur $11 \%$ adlige Seekadetten. In der von 1902 waren es $13 \%$, in der des Jahres 1905 immerhin $14 \%$.

14 S. Demeter, S. 29. Im Jahre 1860 gab es noch $65 \%$ adlige Offiziere; bis 1913 fiel dieser Prozentsatz auf $30 \%$.

15 S. E. O. Volkmann: Soziale Heeresmißstände als Mitursache des deutschen Zusammenbruchs von 1918, in: Das Werk des Untersudhungsausschusses der Verfassungsgebenden Deutschen Nationalversammlung und des Deutschen Reichstags 1919-1930, 4. Reihe, 2. Abt., Berlin, 2. Aufl. 1929, Bd 11/I, S. 22. Das beinahe geschlossen adelige Gardeoffizierkorps ist das Vorbild gewesen an dem sich zunächst preußische, später in schwächerem Maße das gesamte deutsche Offizierkorps innerlich und äußerlich geformt hat. Seine Standesauffassung aber auch seine Berufsauffassung waren maßgebend und haben die bürgerlichen Offiziere völlig durchdrungen.e 
rine kein rein adliges Offizierkorps; die Offizieraspiranten wurden eher auf Grund ihrer Eignung für den Seeoffizierberuf als nach ihrer sozialen Herkunft eingestellt. Das bedeutete aber nicht ipso facto das Ende jeglichen "adligen Geistes", vielmehr brachte der bürgerliche Offizieraspirant, ob im Heer oder in der Marine, seine eigenen Vorstellungen von der "ritterlichen Ehre« in das Offizierkorps mit. Diese Vorstellungen von "Korpsleben" und "Korpsgeist« uferten häufig zu lächerlichen Extremen aus ${ }^{16}$. Heinrich Mann hat dieses Phänomen in Der Untertan glänzend karikiert. Das romantische Bild eines wadligen Rittertums " wurde auch durch Nobilitierung verdienter bürgerlicher Seeoffiziere gefördert, die sich dadurch noch enger mit der Führungsschicht des Staates identifizieren konnten ${ }^{17}$. Obgleich eine Untersuchung dieser Frage den engen Rahmen dieser Dokumentation sprengen würde, sei doch kurz erwähnt, daß ähnlich wie diese Angehörigen des Bürgertums auch viele ostelbische bürgerliche Gutsbesitzer das eben angeführte feudale Streben Ende des letzten Jahrhunderts an den Tag legten ${ }^{18}$.

Weit wesentlicher für diese Dokumentation ist die Tatsache, daß viele Bewerbungen für das Seeoffizierkorps von den Söhnen reicher, wissenschaftlich gebildeter bürgerlicher Familien kamen. Gerade diese Gruppe bedrohte das althergebrachte Vorrecht der zumeist weniger gebildeten und oft ärmeren Bewerber aus Offizier- und Beamtenfamilien - das heißt der sozial erwünschten Aspiranten, "die den bewährten Traditionen der preußischen Armee* entsprachen ${ }^{19}$-, den Offiziernachwudhs aus ihren Kreisen zu stellen. Mehrere Heeresoffiziere hatten diese Gefahr bereits im letzten Jahrzehnt des 19. Jahrhunderts erkannt. Der preußische Kriegsminister, Generalleutnant Karl v. Einem, beschwerte sich darüber, daß die Marine die besten Offzieraspiranten anzog und fügte hinzu: "Die Söhne unser alten Offiziersfamilien wollten einfach nidht mehr dienen, sondern Geld erwerben; die Söhne der reichen Parvenus dagegen wollten Offiziere werden, hauptsächlich aus Eitelkeit ${ }^{20 . "}$

Hier liegt der Kern des Problems. Im größeren Rahmen findet diese Entwidklung eine Parallele in der Auseinandersetzung zwischen der feudal-agrarischen und der bürgerlich-städtischen Lebensauffassung; im kaiserlichen Offizierkorps nahm diese die Form eines Konflikts zwischen wissenschaftlicher Vorbildung (Schulbildung) und "häuslicher Erziehung " an ${ }^{21}$. Demeter bezeichnete diese Auseinandersetzung treffend dahingehend, daß es sich welliptisch um die Anti-

16 S. Rumschöttel, S. 99; Demeter, S. 116-153. Kurz vor Ausbrud des Weltkriegs erklärte Admiral Hugo v. Pohl, geadelter Sohn eines Zahlmeisters, dem Kapitänleutnant Bogislav v. Selchow: "Sie wissen ja garnicht, mein lieber Seldhow, was Sie vor anderen voraushaben, daß Sie einem Uradelsgeschlecht angehören; denn was für Sie Selbstverständlidkeiten sind, dazu brauchen andere unendlichen Schweiß. Und das ist etwas so häßliches; den muß man immer wieder herunter waschen.* Pohl versuchte seinen ganzen Lebensstil dem des •Grand Seigneurs* Admiral Friedrich Graf v. Baudissin anzupassen. BA-Koblenz, Logbuch Bogislav von Seldow, Bd 36, S. 7189.

17 BA-MA, F 3620/PG 68313 Marine-Kabinett. Adelsverleihungen im Allgemeinen, Bd 1, enthält folgende Liste geadelter Seeoffiziere: Knorr (1896); Koester, Müller, Tirpitz, Grumme, Hollmann (1900); Pustau, Bendemann (1901); Hintze, Fischel (1908); Ingenohl (1909); Fischer (1910); Truppel (1911); Schröder, Capelle (1912); Coerper, Pohl, Lans, Karpf, Thomsen (1913); Henkel (1914); und durch den König von Bayern, Hipper (1916).

10 Rumschöttel, S. 99. S. auch H. Rosenberg: Die Pseudodemokratisierung der Rittergutsbesitzerklasse, in: Moderne deutsche Sozialgeschichte, H.-U. Wehler (Hrsg.), Köln, Berlin 1968, S. 287-308.

10 Dok. 1.

20 F. v. Bernhardi: Denkwürdigkeiten aus meinem Leben, Berlin 1927, S. 263. Bernhardis Besprechungen mit Einem sind v. 10. 12. 1904 u. v. 11. 2. 1905. Vgl. Dok. 10. 
these: Rustikalität und Urbanität, Charakter und ‘Geist`, Voluntarismus und Intellektualismus« handle ${ }^{22}$. Dieses Problem, nämlich "Charakter* oder "Bildung «, wissenschaftliche Vorbildung oder soziale Herkunft als erwünschte Qualitäten des Offiziernachwuchses zu berücksichtigen, wurde um die Jahrhundertwende akut, als die Hochseeflotte langsam wuchs und an Bedeutung gewann.

\section{III}

Die Kadettenauswahl wurde von einer dem Inspekteur des Bildungswesens der Marine unterstellten Seekadetten-Annahme-Kommission überwacht. Die Inspektion des Bildungswesens, durch Allerhöchste Kabinetts-Ordre (AKO) vom 26. November 1895 aus der ehemaligen Direktion des Bildungswesens hervorgegangen, war für alle Angelegenheiten des Seeoffiziernachwuchses zuständig, und ihr Direktor war gleichzeitig ständiger Vorsitzender jener Kadetten-Annahme-Kommission. Die Inspektion war zunächst als Immediat-Stellung ausgezeichnet, wurde aber durch AKO vom 30. März 1907 dem Chef der Ostseestation in Kiel unterstellt. Der Inspekteur des Bildungswesens wurde jedoch als ständiger Vorsitzender der umbenannten "Seekadetten-Annahmekommission" bestätigt; in den neuen Verordnungen hieß es, der Inspekteur sernennt die Mitglieder der Seekadetten-Annahmekommission aus den ihm unterstellten Seeoffizieren, er regelt die Tätigkeit dieser Kommission und entscheidet endgültig über die Zulassung zur Laufbahn und die Einstellung als Seekadett « ${ }^{23}$. Diese Umorganisation stellte zweifellos einen gewissen Prestigeverlust für den Inspekteur des Bildungswesens dar, auf dem Gebiet der Kadettenauswahl erfuhr er aber keinerlei Einschränkung seiner Autorität. Er behielt die »endgültige Entscheidung* über die Zulassung zur Seeoffizierlaufbahn in seinen Händen. Seine wirkliche Macht kann nur daran ermessen werden, daß die Seekadetten-AnnahmeKommission keinerlei Protokolle ihrer Sitzungen führte, daß sie eine Begründung ihrer Entscheidungen nicht abgeben mußte, und daß weiter nur äußerst ungewöhnliche Einzelfälle (vgl. Dok. 1-3) außerhalb der Kommission besprochen wurden.

Die Kommission hatte in ihren Entscheidungen allerdings gewissen Grundlinien zu folgen. Am 1. November 1879 hatte Kaiser Wilhelm I. in einer an den Chef der Admiralität, General Albrecht v. Stosch, gerichteten Anweisung bestimmt, daß letzterer nicht zu großen Wert auf die Zabl der Aspiranten legen solle. "Die Hauptsache bleibt hier die Qualität und die innere Zusammengehörigkeit, welche die jungen Leute schon aus ihren Familien-Beziehungen mit in den neuen Beruf bringen, denn das ist das Fundament, aus dem sich später der feste Zusammenhang eines Offizierersatzes sehr viel leichter und sicherer bilden läßt, als aus sich fern stehenden Elementen ${ }^{24}$." Diese Verordnung hinterließ keinerlei Unklarheit in bezug auf den erwünschten Seeoffiziernachwuchs; sie diente dazu, die Homogenität der Offizierkorps im Heer wie in der Marine zu bewahren. Kaiser Wilhelm II. jedoch leitete durch die AKO vom 29. März 1890

22 Demeter, S. 76.

23 BA-MA, F 3302/PG 66706 Marine-Kabinett. Organisation: Bildungswesen der Marine, Bd 1. Im Zeitrahmen dieser Dokumentation gab es folgende Inspekteure des Bildungswesens: Vizeadmiral Oldekop (1900); Admiral v. Arnim (1901-1906); Vizeadmiral Bordsenhagen (1907-1909); Vizeadmiral Coerper (1910-1911); Vizeadmiral v. Dambrowski (1912-1914). Eine eingehende Untersuchung der Einstellung der einzelnen Inspekteure zur Vorpatentierungsfrage würde den Rahmen dieser Dokumentation sprengen. Es kann aber allgemein bemerkt werden, daß sie alle diese Verordnungen ablehnten.

24 Zit. nach Sandhofer, S. 59. 
einen neuen Kurs auf dem Gebiet des Offiziernachwuchses ein. Der ngesteigerte Bildungsgrad " des Volkes erlaube es nun, daß neben dem "Adel der Geburt* nun auch der "Adel der Gesinnung" das Recht für sich in Anspruch nehmen könne, die "Offizier-Aspiranten " der Zukunft zu stellen. Wilhelm II. betrachtete die Söhne aus Offizier- und Beamtenfamilien weiterhin als "Grundpfeiler des Offizierkorps", aber die AKO ernannte nun zu "Träger[n] der Zukunft" außerdem die Söhne nsolcher ehrenwerter bürgerlicher Häuser, in denen die Liebe zu König und Vaterland, ein warmes Herz für den Soldatenstand und christliche Gesittung gepflegt und anerzogen werden ${ }^{25}$. Bald stimmten die führenden Männer von Heer und Marine aber gegen die Erweiterung der gesellschaftlichen Basis des Offizierkorps; in ihren Augen stellte der Erlaß vom 29. März 1890 die Homogenität des Offizierkorps in Frage ${ }^{26}$. Schon vor der Jahrhundertwende äußerte der Kaiser den Wunsch, "daß nach Möglichkeit Söhne von Offizieren aus königstreuen Geschlechtern und aus Familien, deren Name in der Geschichte Preußens oder anderer deutscher Bundesstaaten verzeichnet sind ... mehr wie bisher in die Marine eintreten * ${ }^{27}$ sollten.

IV

In der preußischen Marine des 19. Jahrhunderts reichten für alle Offizieraspiranten Tertianerkenntnisse aus; dagegen verlangte die Kaiserliche Marine Obersekunda-, dann Primareife. Abiturienten von Gymnasien und Realgymnasien wurden als erwünschte Aspiranten bezeichnet, und im Jahre 1882 wurden die Abiturienten der neugegründeten Oberrealschulen dieser Gruppe hinzugefügt ${ }^{28}$. Um die Jahrhundertwende stellten hauptsächlich Portepeefähnriche aus der Lichterfelder Hauptkadetten-Anstalt und Primaner aus den öffentlichen Gymnasien und aus den berüchtigten "Pressen « die Seeoffizieraspiranten ${ }^{20}$. Diese $\mathrm{Zu}$ sammensetzung des Seeoffizierersatzes in bezug auf die wissenschaftliche Vorbildung entsprach nicht den persönlichen Ansichten des neuen Chefs des $\mathrm{Ma}-$ rine-Kabinetts, des Kontreadmirals Georg Alexander v. Müller. Dieser betrachtete es von Anfang an als seine Aufgabe, "den Eintritt von Abiturienten in die Marine zu fördern, den Eintritt von Primanern und Fähnrichen also möglichst einzudämmen “ ${ }^{30}$. Er hoffte ferner, eines Tages das Abiturium als Aufnahmebedingung für alle Seeoffizieraspiranten einzuführen ${ }^{31}$. Besonders die Portepeefähnriche besaßen nur die notwendigste Schulbildung und Müller betrachtete höhere wissenschaftliche Ausbildung nicht als Luxus oder als ein Standeszeichen, sondern als eine dringende Notwendigkeit für den modernen Seeoffizier. Schon die technischen Fortschritte auf dem Gebiet des Schiffsbaus sowie des Artilleriewesens schienen die Forderung nach dem Abitur zu diktieren. Aber

s BA-MA, F. 6082 Reichs-Marine-Amt. Allgemeines Marinedepartement. Ergänzung der Seeoffiziere, $\mathrm{H} .7$.

20 Vgl. W. Deist: Die Armee in Staat und Gesellschaft 1890-1914, in: Das kaiserliche Deutschland. Politik und Gesellschaft 1870-1918, M. Stürmer (Hrsg.), Düsseldorf 1970, S. 321-323. Ein Berliner Zeitungsjunge faßte die AKO folgendermaßen auf: -Neustes Osterjeschenk des Kaisers! Janzer Adel abjeschafft! Allens nur noch Seelenadella Zit. in: G. A. Craig: The Politics of the Prussian Army 1640-1945, Oxford 1955, S. 237.

27 Nach Forstmeier, S. 193.

28 Ebd., S. 191.

20 Ebd., S. 191. Von den 210 Seekadetten der Crew von 1901 hatten $41 \%$ das Abitur, $15 \%$ waren Fähnriche, $38 \%$ besaßen Primareife u. $6 \%$ waren auf Grund eines kaiserlichen Dispens vom Primazeugnis zugelassen. BA-MA, F 6081 Reids-Marine-Amt. Allgemeines Marinedepartement. IX. 4.1. - 4. Ergänzung der Seeoffiziere, H. 6. Denkschr. seitens d. MarineKabinetts (Kapitän z. S. v. Müller) v. 27. 5. 1901.

st S. Dok. 4. 
hier kamen andere, althergebrachte Anschauungen ins Spiel. "Die Fragen der sozialen Herkunft des Offiziers hängen innerlich aufs engste mit dem Problem seiner wissenschaftlichen Vor- und Ausbildung zusammen; es sind konzentrische Kreise ${ }^{\text {s2 }}$ «

In Bayern war das Abitur bereits 1872 als Aufnahmebedingung für Offizieraspiranten eingeführt worden ${ }^{38}$, in Preußen reichte der Widerstand des Landadels, in dem "stenge Ehrbegriffe und gute Formen festgewurzelte Tradition bilden " ${ }^{34}$, aus, um das zu verhindern. Die überwiegende Mehrzahl der Heeresoffiziere lehnte das Prinzip höherer wissenschaftlicher Vorbildung entschieden ab und bestand statt dessen auf der Uberlegenheit einer "häuslichen Erziehung ", in der Hoffnung, der Armee auf diese Weise weiter den Offiziernachwuchs aus den eigenen Reihen stellen zu können ${ }^{35}$.

Diese Haltung fand öfters Billigung durch persönliche Eingriffe des Kaisers. Er gab eine Reihe Kaiserlicher Dispense von der Primareife als Nothilfe zur Annahme derjenigen Offizieraspiranten, die zwar von der gewünschten sozialen Herkunft, dagegen nur mangelhaft vorgebildet waren ${ }^{30}$; der Kaiser führte um die Jahrhundertwende sogar einen Dispens von den Eintrittsexamen zu dem bereits vorhandenen Dispens von der Primareife ein.

Tirpitz als Staatssekretär des Reichs-Marine-Amts sympathisierte mit Müllers Bestrebungen in bezug auf höhere wissenschaftliche Vorbildung ${ }^{37}$, aber die Gegnershaft älterer Seeoffiziere sowie seiner engsten Mitarbeiter hielt ihn von einer öffentlichen Unterstïtzung des Marine-Kabinettchefs $a b^{38}$. Die Inspek-

32 Demeter, S. 80.

28 Rumschöttel, S. 90-91. 1883 u. 1909 bestätigten verschiedene Kommissionen diese Grundsatzentscheidung und das Abitur blieb Eintrittsbedingung in Bayern bis zum Ende der Monarchie.

s4 Vgl. Dok. 3.

35 Diese Einstellung zur Abiturfrage kommt in einem Bericht des Bayerischen Militärbevollmäctigten in Berlin, Generalmajor Frhr. v. Gebsattel, an den Bayerischen Kriegsminister, General Frhr. จ. Horn, v. 20. 10. 1908 über eine Besprechung dieser Angelegenheit im preußischen Militärkabinett am besten zum Ausdrudk: $\rightarrow$ Niemals würde, so wurde mir versichert, das Militärkabinett seine Zustimmung dazu geben, daß in Preußen das Abiturientenexamen als conditio sine qua non gefordert würde, auch wenn es denkbar wäre, den ganzen Ersatz durch Abiturienten dedken zu können ... Es sei zugegeben, daß des Ofteren Söhne von Offizieren, Gutsbesitzern, kurz von Junkern das Abiturium nicht erreichten; diese von der Offizierslaufbahn auszuschließen, würde aber schweres Unrecht und schwere Undankbarkeit sein . . . Es sei ein Unsinn zu behaupten, das Volk habe die Befreiungskriege, habe den Feldzug 70/71 gewonnen, das Volk sei ohne Führer stets und überall eine hilflose Masse, seine Führer aber waren in überragender Mehrzahl die Abkömmlinge der Offiziere Friedrichs des Großen, die preußischen Junker. Es würde aber auch unklug sein, diese jungen Leute zurüdkzuweisen, denn für die Masse der Offiziere brauche man keine Gelehrten ..., sondern Leute mit praktischem Sinn, mit Ehr- und Pflichtgefühl, mit Begeisterung für ihren Beruf, mit einer gewissen Gewandtheit zur Behandlung von Untergebenen, vor allem aber mit dem Herz auf dem rechten Fledk! Niemand werde behaupten können, daß gerade diese Eigenschaften bei den vielleicht weniger glänzend studierenden Sprößlingen der Offiziersfamilien, der Familien des kleinen preuß. Adels, in denen sie seit zwei Jahrhunderten gepflegt und vererbt wurden, in geringerem Maße vorhanden wären, als bei jungen Leuten von unbekannter Herkunft, aber mir vorzüglichen Examens-Resultaten. \& Zit. in: Rumschöttel, S. 129-130. Generalleutnant Dietrich Graf v. Hülsen-Haeseler war Chef des Militärkabinetts zu dieser Zeit; er starb am 14.11. 1908 in Donaueschingen.

se Demeter, S. $93 \mathrm{ff}$.

97 *Unter den Wenigen dafür war von den älteren Offizieren wohl nur der Staatssekretär จ. Tirpitz, der seinen in die Marine eingetretenen Sohn auch erst das Abitur machen ließ.* Der Kaiser... Aufzeidnungen des Chefs des Marinekabinetts Admiral Georg Alexander v. Müller über die Âra Wilhelms II. W. Görlitz (Hrsg.), Göttingen 1965, S. 157 (zit. Müller).

s日 Vgl. Dok. 8. Müller bestätigte später, daß »das Kabinett die treibende Kraft* in der Abiturfrage war, "gestützt auf die Auffassung des Kaisers und die Zustimmung des Staatssekretärs des Reichsmarineamts, der dabei freilich seine Behörde nur sehr teilweise hinter sidh hatte . Müller, S. 49. 
tion des Bildungswesens nahm ebenfalls einen ablehnenden Standpunkt ein ${ }^{90}$. Diese Auseinandersetzung hat mit dazu beigetragen, die Stellung Admirals v. Müller innerhalb des Seeoffizierkorps zu untergraben ${ }^{40}$.

V

Die königlich-preußische Armee hatte durch AKO vom 13. Februar 1900 eine Vorpatentierung um zwei Jahre für alle ihre Offizieraspiranten mit Abitur eingeführt. Die Absicht, diese Verordnung auch auf die Seekadetten der Marine auszudehnen, mußte vom Kaiser wegen Widerstandes »des größten Teils des Seeoffizierkorps« aufgegeben werden ${ }^{41}$. Die Verordnung für die Marine war unzureichend vorbereitet worden, und Admiral v. Müller sah ein, daß die Vorpatentierungs-Reglements der Armee, das heißt Vorpatentierung bereits mit dem Offizierspatent, nicht einfach von der Marine übernommen werden konnten ${ }^{42}$. Jedoch gab Müller seine Pläne nicht auf. Im Frühjahr 1907 griff er die Vorpatentierungsfrage erneut auf und war sich dabei bewußt, daß große Teile des Seeoffizierkorps seine Ansichten nicht teilten. Er hielt jedoch nach wie vor an seiner Uberzeugung fest, daß nur höhere wissenschaftliche Bildung die gehobene soziale sowie militärische Stellung des Seeoffizierkorps begründen und erhalten konnte. Müller betrachtete das gegenwärtige System, wonach der Abiturient freiwillig zwei Jahre in der Anciennität einbüßte, als "unmoralisch», und er gab offen zu, daß in Zukunft das Abitur als Aufnahmebedingung eingeführt werden sollte ${ }^{43}$. Er bat Admiral v. Tirpitz die Vorpatentierung zu unterstützen, um durch sein Eintreten für die Sache der Verordnung größeres Gewicht

30 Am 12.1.1912 unterrichtete der Inspekteur des Bildungswesens, Admiral Carl Coerper (S. Anm. 17) den Staatssekretär: »Ih bin mir vollkommen darüber klar: auf der einen Seite steht das große allgemeine und dringende Interesse der gesamten Marine an der Beseitigung des latenten Offiziermangels und an der Schlagfertigkeit und Ms. Bereitschaft bezüglich des Offizierpersonals, auf der anderen Seite das Interesse des Offizierkorps - aber doch auch des Dienstes - an einem Offiziernadwuchs von guter häuslicher Erziehung, vornehmer Gesinnung, guter Familientradition und sicherem Auftreten, und bin mir nicht im Zweifel, daß in

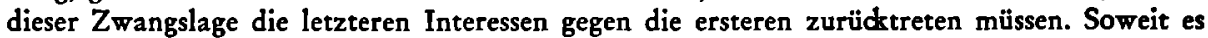
aber dringend noch möglich ist, und besonders auch mit Rüdksicht auf die oben geäußerten Bedenken muß die Seekadetten-Annahme-Kommission versuchen, auch den letztgenannten Interessen noch tunlichst Rechnung zu tragen. * BA-MA, F 3356/PG 66977, Bd 1. Coerper war in seiner Eigenschaft als Vorsitzender der Seekadetten-Annahme-Kommission in der Lage, seine Ansichten durchzusetzen.

10 Nach dem Krieg erklärte Vizeadmiral Eberhard v. Mantey, „daß die Personalpolitik in der Marine von Grund auf falsch war. Mit der Hürde des Abiturientenexamens fängt die Geschichte an*. BA-MA, F 7590, Nachlaß Hollweg, Bd 4. Mantey an Vizeadmiral a. D. Karl Hollweg, 16. 4. 1929. Vizeadmiral Paul Wurmbach, von 1917 bis 1918 Inspekteur des Bildungswesens der Marine, beschwerte sich später über die Nur-Abiturienten-Marotte* eines Kabinettschefs, der "als soldher unserer Marine in einem Jahr mehr geschadet hatte, als sich in zehn wieder gut machen ließe. Hatte unsere Marine denn mit den Nichtabiturienten so schlechte Erfahrung gemacht? Scheer, Graf Spee, Tirpitz, Koester, Deinhard?!* BA-MA, Nachlaß Behndke, N 173, Bd 8, S. 48. Wurmbach an Behndke, 23.11. 1921. Wurmbach sprach sich gegenüber Behndse gegen das Abitur als Eintritesbedingung für den Offiziernachwuchs der Reichsmarine aus.

4 BA-MA, F 6081, H. 6. Marine-Kabinett an Tirpitz, 19. 3. 1902.

42 Müller erklärte später, daß bei der kurzen Leutnantszeit in der Marine (ungefähr 2 Jahre) - der eben zum Leutnant beförderte Abiturient schon in Jahresfrist vor der Beförderung zum Oberleutnant gestanden * hätte. Dies wäre *ein Unding * gewesen, denn der Oberleutnant z. S. habe als Wachhabender Offizier* eine äußerst verantwortungsvolle Stellung eingenommen. Der Abiturient habe 2 Jahre wissenschaftliche Vorbildung mehr, aber andererseits 2 Jahre praktische Ausbildung weniger als der Fähnrich z.S. besessen. Dementsprechend habe er, Müller, die Vorpatentierung der Abiturienten für die Zeit der Beförderung zum Oberleutnant z.S. oder sogar erst zum Kapitänleutnant festgesetzt, "zu welchem Zeitpunkt die technische Ausbildung als einigermaßen ausgeglichen gelten konnte«. Müller, S. 49, 157.

is Dok. 4. 
zu verleihen. Der Staatssekretär lehnte jedoch ab ${ }^{44}$. Müller bereitete den Erlaß also selber vor, und am 2. Juli 1907 unterzeichnete Wilhelm II. eine AKO, die - angefangen mit der Crew von 1909 - für alle Abiturienten, deren Charakter außer Frage stand, und die ihre praktische Ausbildung und ihre Seeoffizierprüfung mit "gut" bestanden hatten, eine Vorpatentierung um ein Jahr in Aussicht stellte ${ }^{45}$. Das Vorrücken in der Anciennität sollte zur Zeit der Beförderung zum Oberleutnant z. S. erfolgen.

Die meisten Seeoffiziere reagierten auf diese AKO negativ. Diese allgemeine Kritik fand in dem Chef der Hochseeflotte, Admiral Prinz Heinrich von Preußen, einen einflußreichen Fürsprecher ${ }^{46}$. Sein Bericht vom 17. März 1908 (Dok. 6), basierend auf Berichten aller Schiffskommandanten, ist exemplarisch für die allgemeine Beweisführung gegen das Abitur als Aufnahmebedingung. Die AKO würde nur "Zwiespalt und Unzufriedenheit " sowie zu viel reine Theorie in das Seeoffizierkorps einführen. Es würden sich durch diese Verordnung zwei verschiedene Klassen von Offizieren, "die sich feindlich gegenüberstehen ", entwikkeln. Die Vorpatentierung der Abiturienten würde sofort einen Mangel an Bewerbern erzeugen, und die Söhne von werwïnschten Elementen «, bei denen "Vererbung, Rasse und Erziehung " zusammenträfen, vom Seeoffizierkorps fern halten. Soldh ein Verlust an Nachwuchs aus den walten guten Offiziers- und Beamtenfamilien « würde die Marine zwingen, ihre Offizieraspiranten aus anderen sozialen Schichten zu holen, "deren Söhne vielleicht eine höhere wissenschaftliche Bildung, aber weder die Erziehung noch die sittlichen und sozialen Anschaunngen mitbringen, die jene verbürgen*. Und je mehr Aspiranten aus den weniger erwünschten Kreisen kämen, desto größer würde die Abneigung in den erwünschten sozialen Kreisen werden, ihre Söhne zur Marine zu geben. Es sei überhaupt für die junge, traditionsarme Marine höchst notwendig, ihren Nachwuchs aus anerkannten Offizier- und Beamtenfamilien zu rekrutieren, um die "allmählich sich bildenden Traditionen und Rasseeigenschaften " weiter zu fördern. Zum Schluß bemerkte der Prinz noch, daß der Ausfall der Auslandsreisen einen äußerst nachteiligen Einfluß auf den Seeoffiziernachwuchs ausübe ${ }^{47}$. Admiral v. Müller lehnte diese scharfe Kritik entschieden ab ${ }^{48}$. Weder Müller noch Wilhelm II. wollten das soziale Niveau des Seeoffizierkorps herabsetzen, aber beide Männer sahen klar voraus, daß das moderne Zeitalter der Technik und der Wissenschaft sowie der beabsichtigte Umfang der Tirpitz'schen Schlachtflotte und die dazu benötigte Anzahl an qualifizierten Seeoffizieren keine andere Wahl mehr ließen, als die Seeoffizierkarriere in Zukunft den Söhnen des Bildungs- und Besitzbürgertums in verstärktem Maße zu öffnen ${ }^{49}$. Müller sprach sich gegen die von Tirpitz vorgeschlagene worganische Entwidklung «, das heißt die Hoffnung, daß sich die ganze Sache mit der Zeit selbst klären werde ${ }^{50}$, aus und beharrte fest auf seiner Auffassung, daß nur höhere wissenschaft-

4 Dok. 4 n. Anm. 90.

15 Dok. 5.

40 Müller beschrieb den Prinzen Heinrich später als den "Sprecher der Ablehnenden*. "Der Kaiser erzählte mir das cinmal bei einem Vortrag. Der Prinz Heinrich habe ihm gesagt, der Admiral v. Müller ruiniere mit seiner `Bevorzugung der Abiturienten ... das ganze Offizierkorps, es meldeten sich nur Schlachtersöhne usw.« Müller, S. 157-158.

(7) Dok. 6.

6. Vgl. Dok. 7.

4 Müller erklärte später, daß sich der Kaiser nicht durch Prinz Heinrichs ablehnende Stellungnahme irre machen ließ, "wohl wissend, $\mathrm{da} B$ in der die Standesunterschiede überbrückenden neuen Zeit die höhere Allgemeinausbildung eine immer wichtiger werdende Bedingung für Ausübung einer Autorität geworden ist๔. Müller, S. 158.

so Dok. 8 . 
liche Bildung die Führungsrolle des Seeoffizierkorps innerhalb der Marine gewährleisten könne, und daß das Abitur über kurz oder lang als Aufnahmebedingung für alle Seeoffizieraspiranten eingeführt werden müsse ${ }^{\mathbf{5 1}}$.

VI

Was waren die unmittelbaren Ergebnisse der AKO vom 2. Juli 1907? Die Frage ist zum Teil akademisch, da der Krieg diese Entwicklung unterbrach 52. Der Prozentsatz der Abiturienten unter den Seekadetten nahm seit 1907 ständig zu, wie die folgende Tabelle ${ }^{53}$ zeigt:

$\begin{array}{lllrcl}\text { Jabrgang } & \text { Crewgröße } & \begin{array}{l}\text { Portepee- } \\ \text { fähnriche }\end{array} & \text { Primaner } & \text { Abiturienten } & \begin{array}{l}\text { Prozentsatz } \\ \text { Abiturienten }\end{array} \\ 1898 & 107 & 18 & 66 & 23 & 21 \\ 1900 & 203 & 26 & 105 & 72 & 35 \\ 1903 & 150 & 24 & 54 & 72 & 48 \\ 1905 & 159 & 26 & 45 & 88 & 55 \\ 1907 & 189 & 31 & 53 & 105 & 55 \\ 1909 & 202 & 12 & 33 & 157 & 77\end{array}$

Zur gleichen Zeit ging die Anzahl der Primaner und Fähnriche bei zahlenmäßig stärkerem Jahrgang bedeutend zurück. Bereits am 4. Dezember 1907 unterrichtete die Inspektion des Bildungswesens der Marine Admiral v. Müller, daß weniger Meldungen eingegangen seien, daß fast $80 \%$ aller Seeoffizieraspiranten das Abitur besaßen, und daß diese Aspiranten "in socialer Beziehung " vieles zu wünschen übrig ließen. "Es haben sich viele junge Leute aus skleinen، Familien gemeldet, und die ersten Kreise melden ihre Söhne nicht an, wenn sie wissen, daß wir jene annehmen ${ }^{54 . " ~ E i n e ~ U n t e r s u c h u n g ~ d e r ~ B e r u f e ~ d e r ~ V a ̈ t e r ~ v o n ~}$ den 96 der Crew von 1906 angehörigen Abiturienten zeigt, daß 24 Söhne von Akademikern und Pastoren, 25 von Kaufleuten, Fabrikbesitzern und Fabrikpächtern und 9 sogar Söhne von Unteroffizieren waren. $60 \%$ der Abiturienten kamen also aus den sozial weniger erwünschten Gesellschaftskreisen. Söhne von Offizieren nicht unter dem Oberstenrang und von Staatsbeamten mit wenigstens dem Titel des Rats II. Klasse wurden als erwünschter Offizierersatz bezeichnet ${ }^{\mathbf{5 5}}$. Die Gefahr bestand, daß die Inspektion des Bildungswesens von nun an wissenschaftliche Vorbildung mit unerwünschter sozialer Herkunft gleichsetzte. Dieses qualitative Dilemma wurde ferner durch einen rein quantitativen Druck verstärkt.

Im Jahre 1902 bestand das kaiserliche Seeoffizierkorps aus 194 höheren Seeoffizieren (Korvettenkapitän bis Admiral), weiteren 821 Seeoffizieren (Leutnant z. S. bis Kapitänleutnant I. Klasse) und 601 Seeoffizieraspiranten (Seekadetten und

51 Dok. 9.

32 * Praktisch ist die Frage der Vorpatentierung übrigens gar nicht mehr geworden. Der Krieg kam dazwischen.\& Müller, S. 158. Die Beförderung der Angehörigen der ersten (1909) Crew zum Oberleutnant z.S. und die Vorpatentierung der Abiturienten waren frühestens Ende 1914/Anfang 1915 zu erwarten. Nach dem Krieg wurde von der Reichsmarine das Abitur als Eintrittsbedingung verlangt.

53 BA-MA, F 6082, H. 8. Admiral Coerper an das Reichs-Marine-Amt, 16. 3. 1910. Vgl. Anm. 29.

- BA-MA, F 3548/PG 68066 Marine-Kabinett. Bestimmung über Fähnrichs zur See und Seekadetten, Bd 1. Korvettenkapitän Kurt Frhr. v. Rössing, Adjutant des Inspekteurs des Bildungswesens der Marine (Vizeadmiral Ludwig Bordkenhagen) an Vizeadmiral v. Müller, 4. 12.1907.

s5 BA-MA, F 6082, H. 7. Anl. 8 u. 9 einer Reichs-Marine-Amt Denkschr. v. 1907. 
Fähnriche z. S.) ${ }^{56}$. Fünf Jahre später standen 249 höhere Seeoffiziere 1286 jüngeren Seeoffizieren und 553 Seeoffizieraspiranten gegenüber ${ }^{57}$. Und 1913 bestand das 2249 Mann starke Seeoffizierkorps aus 385 höheren Seeoffizieren, 1811 jüngeren Seeoffizieren und 643 Seeoffizieraspiranten ${ }^{38}$. Obgleich die Vergrößerung des Seeoffizierkorps nach Proportionsprozenten in den drei angedeuteten Gruppen gleichmäßig stieg (von 194 höheren Seeoffizieren im Jahre 1902 auf 385 im Jahre 1913, gegenüber 821 jüngeren Seeoffizieren 1902 und 1811 im Jahre 1913), ist doch festzustellen, daß, rein numerisch gesehen, die Anzahl der jüngeren Seeoffiziere gegenüber der Zahl der höheren Seeoffiziete enorm zugenommen hatte. Werden noch die Seeoffizieraspiranten der Gruppe der jüngeren Seeoffiziere hinzugerechnet, standen im Jahre 1913385 höhere Seeoffiziere 2454 jüngeren Mitgliedern des Seeoffizierkorps gegenüber. Weitaus die meisten jüngeren Seeoffiziere und Seeoffizieraspiranten besaßen das Abitur, was unter den höheren Seeoffizieren durchaus nicht der Fall war ${ }^{59}$. Dementsprechend war es kein Wunder, daß viele ältere Seeoffiziere am Ende des ersten Jahrzehnts des 20. Jahrhunderts bezüglich der sozialen Zusammensetzung des Korps sowie in der Frage der erwünschten wissenschaftlichen Vorbildung der Seekadetten eine gewisse Unruhe und Unzufriedenheit zeigten; es handelt sich hier jedoch um einen sich anbahnenden, keineswegs grundsätzlichen Konflikt zwischen einer älteren und einer jüngeren Führungsschicht.

Wie weit diese Unzufriedenheit bereits vor dem Krieg ging, zeigt ein Bericht des Inspekteurs des Bildungswesens der Marine, Vizeadmiral Hans. v. Dambrowski, vom 7. April 1913 an den Staatssekretär des Reichs-Marine-Amts ${ }^{\text {00. Dambrow- }}$ ski kritisierte jegliche Bevorzugung oder Vorpatentierung von Abiturienten, da ein derartiges Vorgehen nur zu dem "unerwünschten Ziel « führen könne, daß der Nachwuchs sich vornehmlich aus "mittelmäßigen Abiturienten " und "schlechten Primanern" zusammensetzen würde. Der Inspekteur befürwortete zwar die Annahme von "aus erwïnschten Kreisen kommenden Abiturienten “, aber er bevorzugte eine »unentbehrliche gesellschaftliche Mischung: Land, Armee, höhere Staatsbeamte neben den gut bürgerlichen Kreisen “. Er verglich die gegenwärtigen Zustände mit einem "rollenden Stein « und lehnte Müllers Konzept einer höheren wissenschaftlichen Vorbildung ab. "Ein Offizierkorps dessen Bewertung darin gipfelt, daß es in allen seinen Gliedern über die abgeschlossene

so Höhere Seeoffiziere: 3 Admirale, 4 Vizeadmirale, 11 Kontreadmirale, 57 Kapitäne und 119 Fregatten- u. Korvettenkapitäne. Jüngere Seeoffiziere: 128 Kapitänleutnante I. Klasse, 92 Kapitänleutnante II. Klasse, 348 Oberleutnante u. 253 Leutnante. Seeoffizieraspiranten: 401 Fähnriche z. S. u. 200 Seekadetten. BA-MA, F 6513. Etat für die Verwaltung der Kaiserlichen Marine auf das Rechnungsjahr 1902, S. 26-28, 155. Diese Liste enthält auch Angaben über die verabschiedeten Seeoffiziere, gibt aber keinerlei Informationen bezüglich der wenigen $\gg$ z. D.* gestellten Seeoffiziere in aktiven Dienststellen. Für unsere Zwecke sind diese wenigen Ausnahmen ohne Bedeutung.

37 Höhere Seeoffiziere: 4 Admirale, 7 Vizeadmirale, 15 Kontreadmirale, 74 Kapitäne u. 159 Fregatten- u. Korvettenkapitäne. Jüngere Seeoffiziere: 220 Kapitänleutnante I. Klasse, 159 Kapitänleutnante II. Klasse, 503 Oberleutnante u. 404 Leutnante. Seeoffizieraspiranten: 378 Fähnriche z. S. u. 175 Seekadetten. BA-MA, F 6514, S. 26, 166.

s8 Höhere Seeoffiziere: 4 Admirale, 10 Vizeadmirale, 20 Kontreadmirale, 92 Kapitäne u. 259 Fregatten- u. Korvettenkapitäne. Jüngere Seeoffiziere: 533 Kapitänleutnante u. 1278 Oberleutnante u. Leutnante. Seeoffizieraspiranten: 398 Fähnriche z. S. u. 245 Seekadetten. BA-MA, F 6515, S. 24, 139.

80 Eine Marine-Kabinetts Denkschr. v. September 1907 zeigt, daß 23,8\% der Kapitäne und 50,9\% der Fregatten- u. Korvettenkapitäne (43,6\% Durchschnitr) Abiturienten waren. Demgegenüber waren 55,8\% der Kapitänleutnante u. 69,6\% der Oberleutnante Abiturienten. BA-MA, F 3470/PG 67631 Marine-Kabinett. Qualifikationsliste über Seeoffiziere, Bd 3. Die meisten Admirale waren Nidhtabiturienten.

oo Für die folgenden Zitate vgl. Dok. 10. 
Schulbildung verfügt, dies aber auf Kosten der ritterlichen und traditionellen Eigenschaften des deutschen Offizierstandes, ist für die Marine nicht erstrebenswert. Es wird nach innen und nach außen versagen." Dambrowski beklagte darüber hinaus den Geburtenrückgang in den erwünschten Gesellschaftskreisen; ferner bemühten sich die Söhne aus Offizier- und Beamtenfamilien allzuoft um Stellungen mit besserer Bezahlung als sie der Offizierberuf bieten konnte. Als Folge dieser Entwicklung werde die Marine gezwungen, andere, weniger erwünschte soziale Gesellschaftsschichten heranzuziehen. "Je mehr von unten zufließt, desto mehr fließt von oben ab. « Die Vorpatentierung bringe einen weiteren $Z$ wiespalt in das Korps. Dambrowski sprach wie Prinz Heinrich fünf Jahre früher erneut von der Entwicklung zweier verschiedener Klassen von Seeoffizieren, der mit und der ohne Abitur. "Meiner festen Überzeugung nach ist die Vorpatentierung der springende Punkt, das srote Tuch, das viele erwünschte Elemente zurückschredkt. Nur von ihrer vollkommenen Beseitigung wird man eine wesentliche Besserung erwarten dürfen. « Der Schaden, der durch die AKO vom 2. Juli 1907 bereits entstanden sei, vergrößere sich von Jahr zu Jahr »und zwar in potenzierter Form «. Am Ende des Berichts kam der Inspekteur des Bildungswesens zu der Schlußfolgerung: "Denn an der Tatsache wird nicht mehr gezweifelt, daß wir uns in sozialer Richtung in einer Abwärtsbewegung befinden.* Am 5. Mai 1913 nahm der Direktor der Marineschule in Mürwik, Kapitän z. S. Herwarth Schmidt v. Schwind, Stellung zu Dambrowskis Kritik. Schwind stimmte Dambrowskis Urteil über die Vorpatentierungsfrage völlig zu und beklagte auch, daß der Seeoffizierersatz in sozialer Hinsicht vieles zu wünschen übrig lasse. "Bleibt alles beim Alten, so müssen wir in Bezug auf die Beschaffung geeigneten Ersatzes uns Bankrott erklären "1. "Ein Jahr später zog das Reich mit diesem Seeoffizierkorps in den Krieg.

VII

Der von Prinz Heinrich beklagte Mangel an Auslandsreisen hing innerlich mit dem Tirpitz'schen Flottenplan zusammen; der Schwerpunkt der deutschen Flottenpräsenz lag in zunehmendem Maße nach dem China-Unternehmen von 1900/ 01 in der Nord- und Ostsee, die Zahl der Auslandskommandos nahm ab ${ }^{\mathbf{0 2}}$. Diese Entscheidung brachte auch auf dem Gebiet der Personalpolitik gewisse Nachteile, denn die Anziehungskraft auf den erwünschten Nachwuchs ließ nach ${ }^{63}$. Diese Beschränkung auf Fahrten in europäischen Gewässern übte zudem einen nachteiligen Einfluß auf den von älteren Seeoffizieren mehrfach angeführten *weltoffenen « und "weltgewandten “ Geist des Seeoffizierskorps aus. Nach dem Krieg erklärte Vizeadmiral Eberhard v. Mantey: »Man tanzte mit irgendeiner Übungsflotte eine Polonaise zwischen Zoppot und Kiel und damit war Schluß ot.«

Um die Jahrhundertwende hatte die Kaiserliche Marine gegenüber der königlichpreußischen Armee die größere Bedeutung in Fragen der Außen- sowie der Innenpolitik erreicht ${ }^{05}$. Diese Entwidklung, noch unterstïtzt durch das Streben der Seeoffiziere, die soziale und militärische Gleichstellung mit dem Offizierkorps

(1) BA-MA, F 6082, H. 10.

a2 S. P. M. Kennedy: Tirpitz, England and the Second Navy Law of 1900: A Strategical Critique, in: MGM 2/70, S. 38 (zit. Kennedy).

os Vgl. Dok. 6 u. Anm. 105.

o4 BA-MA, F 7590, Nachlaß Hollweg, Bd 4. Mantey an Hollweg, 16. 4. 1929.

or S. dazu V. R. Berghahn: Zu den Zielen des deutschen Flottenbaus unter Wilhelm II., in: HZ 210 (1970), H. 1, S. 34-100 (zit. Berghahn) sowie Berghahn u. Deist: Kaiserliche Marine und Kriegsausbruch 1914. Neue Dokumente zur Juli-Krise, in: MGM 1/70, S. 37-44. 
der Garde-Regimenter zu erreichen, machte es besonders notwendig, eine äußerst starke Einflußnahme auf die Auswahl der Seeoffizierbewerber auszuüben. Nur in diesem Zusammenhang ist es verständlich, daß ältere Seeoffiziere von einem "dringenden Mangel " an geeigneten Bewerbern sprechen konnten zu einer Zeit, in der die Armee klagte, daß die besten Offizieraspiranten zur Marine gingen. Denn man sprach offensichtlich nicht von einem quantitativen, sondern von einem qualitativen Mangel. Die von Admiral v. Müller bevorzugte höhere wissenschaftliche Vorbildung (Abitur) als Aufnahmebedingung ließ die Zahl der Bewerbungen nicht zurüdkgehen, verschaffte aber Bewerbern aus weniger erwünschten sozialen Gesellschaftsschichten vermehrt auf Kosten der Aspiranten aus Offizier- und Beamtenfamilien Eintritt in die Laufbahn. Die folgende Tabelle ${ }^{66}$ bestätigt diese Feststellung:

$\begin{array}{llllll}\text { Jahrgang } & \text { Bedarf } & \text { Anmeldungen } & \text { Eingestellt } & \text { Abitur } & \begin{array}{l}\text { Prozentsatz } \\ \text { mit Abitur }\end{array} \\ 1900 & 200 & 415 & 203 & 72 & 35 \\ 1902 & 200 & 411 & 200 & 92 & 46 \\ 1905 & 160 & 319 & 159 & 88 & 55 \\ 1907 & 190 & 372 & 189 & 105 & 55 \\ 1908 & 200 & 329 & 201 & 155 & 77 \\ 1910 & 230 & 372 & 205 & 176 & 86 \\ 1911 & 230 & 424 & 213 & 182 & 85 \\ 1912 & 260 & 448 & 242 & 202 & 83 \\ 1913 & 315 & 526 & 289 & 206 & 71\end{array}$

Ein quantitativer Mangel an Bewerbern bestand ganz einfach nicht. Ihre Zahl stieg seit 1909, dem Jahr, in dem die AKO vom 2. Juli 1907 in Kraft trat, ständig. Seitdem ist auch eine bedeutsame Zunahme im Prozentsatz der Bewerber mit Abitur festzustellen. Dementsprechend kann nur in bezug auf die erwünschte soziale Herkunft von einem "Mangel k an geeigneten Bewerbern die Rede sein. Schließlich ist es interessant, daß in dem Jahr (1913), in dem Admiral v. Dambrowski von der Inspektion des Bildungswesens und Kapitän v. Schwind von der Marineschule sich auf das entschiedenste gegen Müllers Vorpatentierungskonzept wandten, der Prozentsatz der Abiturienten unter den Seeoffizieraspiranten von $83 \%$ auf $71 \%$ zurückging. Es kann kaum bezweifelt werden, daß diese Abnahme direkt auf die ablehnende Stellungnahme von Schwind und von Dambrowski, der schließlich die endgültige Entscheidung in dieser Angelegenheit traf, zurüdkzuführen ist. Letzten Endes waren es nur Müllers Festigkeit und die Unterstiitzung durch den Kaiser, die bereits im Jahre 1914 den Prozentsatz von Abiturienten auf $90 \%$ ansteigen ließen ${ }^{67}$.

Hermann Rumschöttel hat in seiner glänzenden Arbeit gezeigt, daß sich das Abiturium in Bayern zu einem "Bildungsprivileg" sowie zu einer "Bildungsschranke» entwidkelte, indem Adel und Bürgertum höhere wissenschaftliche Vorbildung gemeinsam dazu benützten, unerwünschte soziale Elemente vom Offizierkorps fern zu halten ${ }^{68}$. Die Mehrzahl des höheren Seeoffizierkorps vor 1914 als Repräsentant der Kaiserlichen Marine, die das Lieblingskind des Bildungs- und Besitzbürgertums war, lehnte dagegen höhere wissenschaftliche Vor- 
bildung (Abitur) gegenüber "häuslicher Erziehung " als Aufnahmebedingung für die Seeoffizierlaufbahn ab.

Der Konflikt über soziale Herkunft oder höhere wissenschaftliche Vorbildung als erwünschte Eigenschaften für den Eintritt in die Seeoffizierlaufbahn ist in der Kaiserlichen Marine nie gelöst worden. Gemäßigte Kompromisse brachten keine Lösung. Die Marine erreichte nicht die wünschenswerte Synthese zwischen den scheinbar unversöhnlichen Polen höherer wissenschaftlicher Vorbildung und „häuslicher Erziehung ; dieser Konflikt führte zu einem $Z$ wiespalt innerhalb des kaiserlichen Seeoffizierkorps. Die Inspektion des Bildungswesens und vor allem ältere Seeoffiziere bildeten mit Prinz Heinrich von Preußen eine scharfe Front gegen Admrial v. Müllers modernes Bildungskonzept. Der Kaiser unterstützte Müllers Bestrebungen im Prinzip ${ }^{\circ 9}$ und bevorzugte hohe Ausbildungskosten als indirekte Schranke gegen die Gefahr, daß zu viele sozial unerwünschte Elemente in das Seeoffizierkorps kommen könnten ${ }^{70}$. Admiral v. Tirpitz nahm eine schwankende Haltung ein. Kurz vor Ausbruch des Krieges waren zwei der zuständigen Marineinstanzen (Inspektion des Bildungswesens und Marineschule) sogar davon überzeugt, daß die Kaiserliche Marine in ihrer Personalpolitik bereits "Bankrott" erlitten hatte. Holger H. Herwig

- Am 26. 7. 1909 unterrichtete der Kaiser Admiral v. Müller, daß er Abiturienten als den *bevorzugten Ersatz des Seeoffizierkorps* betrachtete. Wilhelm II. richtete Müllers Aufmerksamkeit auf das niedrige wissenschaftliche Niveau der Lichterfelder Kadetten und befahl Müller, keine wbesondere Rüdssicht« auf diese Kadetten, die nur $6 \%$ des Seeoffiziernachwuchses bildeten, zu nehmen. Berichte der Shulschiffskommandanten hatten eindeutig bestätigt, daß die praktische Ausbildung der Abiturienten sich »durchweg besser* vollziehe als die der Nidhtabiturienten. BA-MA, F 6082, H. 9. Müller an Tirpitz, 26. 7. 1909.

70 Vgl. Dok. 3. Eine Arbeit des Marineingenieurs Otto Dittrich v. Marine-Kabinett schätzte im Jahre 1910 die Familienzuschüsse eines Seeoffizieraspiranten bis zur Beförderung zum Oberleutnant z. S. auf 8000 M. BA-MA, F 3356/PG 66969 Marine-Kabinett. Marine Ingenieure, Bd 4. K. Peter: Seeoffizieranwärter-Ausbildung in Preußen/Deutschland von 1848-1945, Ms in: BA-MA, III M (A) 50, S. 130-131, setzt den Familienzuschuß um 1909 auf 9755 M an. 
1. Admiral und Inspekteur des Bildungswesens der Marine Volkmar v. Arnim an den Kontreadmiral und Chef des Marine-Kabinetts Georg Alexander v. Müller, 1. 2. 1907. $B A-M A, F$ 3356/PG 66977. Kais. Marine-Kabinett. Bestimmungen über Einstellungen. $B d 1$.

Euer Hochwohlgeboren beehre ich mich in Beantwortung des sehr gefälligen Schreibens vom 31. Januar $1907^{71}$ das Nachstehende ganz ergebenst zu erwidern:

Die Seekadetten-Annahme-Kommission ist stets von dem Grundsatze ausgegangen, im Interesse des Offizierkorps und zum Besten der Marine, diejenigen jungen Leute als Seekadetten-Anwärter anzunehmen, die den bewäbrten Traditionen der preussischen Armee 72 entsprechend eine Gewähr dafür bieten, dass sie in jeder Beziehung den hohen Anforderungen entsprechen, die an den Offizierstand gestellt werden.

Ist der Bedarf gross und gehen die Anmeldungen spärlich ein, so wird man, wie dies wäbrend einer Reibe von Jahren nibht zum Besten der Marine ${ }^{78}$ nötig war, junge Leute einstellen müssen, die den zu stellenden Anforderungen nur unvollkommen entsprechen. In diesem Jahre liegen die Verhältnisse umgekehrt. Eine Reibe von Bewerbern ${ }^{74}$ muss nur aus dem Grunde abgewiesen werden, weil der Bedarf gedeckt ist. Die SeekadettenAnnahme-Kommission hält es für ibr selbstverständliches Recht und für ihre Pflicht, die Geeignetsten auszuwählen. Sie ist nicht in der Lage auf die zahlreichen und schriftlichen Empfehlungen ${ }^{75}$ ein besonderes Gewicht zu legen.

Söhne von Feuerwerksoffizieren ${ }^{70}$ und in ähnlichen sozialen und gesellschaftlichen Verhältnissen lebenden Familien halte ich ohne weiteres nicht für eine wünschenswerte Aquisition für unser Seeoffizierkorps. Man wird sie nur annehmen, wenn es an geeigneteren Bewerbern fehlt ${ }^{\text {? }}$.

Auch bezweifele ich, dass die Armee auf die Gewinnung von jungen Leuten aus diesen Schichten der Bevölkerung besonderen Wert legt; sie ist gezwungen. sie zu nehmen, um dem herrschenden Mangel zu begegnen.

Zum Schluß beehre ich mich noch Euer Hochwohlgeboren Aufmerksamkeit auf eine Allerhöchste Willensäusserung zu lenken, dass Seine Majestät grundsätzlich nicht beabsichtigen in die Befugnisse der Seekadetten-Annahme-Kommission einzugreifen.

Schreiben des Chefs des Marinekabinetts vom 2. Januar $1904{ }^{78}$.

von Arnim

71 Zum Schreiben Müllers vgl. Anm. 76, vgl. auch Brief Arnims v. 25.1.1907 an den Kaiser betr. Dworadks Ablehnung "mit Rüdksicht auf die Familienverhältnisse und die soziale Stellung des Gesuchsstellers. Dworads ist Subalternbeamter. Auch bieten die Vermögensverhältnisse keine Gewähr für die Zahlung der monatlichen Zulage.* BA-MA, F 3356/PG 66977.

72 Von Müller unterstrichen, mit der Randbemerkung: »diese Traditionen lassen es doch zu, daß den Söhnen von F. Werks Off. der Eintritt i. d. Armee durch das Kadet.Korps sogar erleidhtert wird..

23 Von Müller unterstrichen, mit der Randbemerkung: „Bitte festzustellen, aus welchen Kreisen die üblen Elemente im Seeoffizierkorps während der letzten 10 Jahre kommen (Ehrenger. u. Kriegsger. Abgänge).*

it Von Müller unterstrichen, mit der Randbemerkung: *dann ist es doch auffallend, daß ... [?] so vielen der Dispens vom Prima Zeugnis befürwortet wird. Wieviel sind es? *

75 Vgl. Dok. 2.

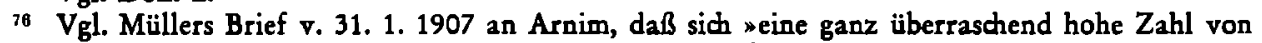
Söhnen von Feuerwerksleutnants im Lichterfelder Kadetten-Korps befinden. •Die Armee hat sich offenbar mit der Notwendigkeit abgefunden, auch diese Kreise, in die wohl der Gefängnisinspekteur mit herein zu nehmen sein würde, zurüdkzugreifen. Ob die Marine hierin einen anderen Standpunkt einnehmen soll z. B. auch gegenüber den etwa aus dem Kadettenkorps sich zur Marine meldenden Söhnen von Feuerwerk. Offizieren, ist eine Frage, die in dem Falle Dworads kaum umgangen werden kann.* BA-MA, F 3356/PG 66977.

i) Müllers Randbemerkung: „So einfach liegt die Sache denn doch nicht. Es ist eine große soziale, keine nur gesellschaftliche Frage. Auch kann ich solche Anwärter nicht in einem Jahr annehmen, im nächsten ablehnen.«

78 Bezieht sich auf einen Brief d. Chefs d. Marine-Kabinetts, Gustav Frhr. v. Senden-Bibran, v. 2. 1.1904 an Helene Baronin v. d. Redke betr. Ablehnung ihres Gesuchs, ihren Sohn, trotz erfolgter Ablehnung durch Admiral v. Arnim wegen ungünstigen Charakterzeugnissen von lokalen Dienststellen, als Seekadett in die Marine eintreten zu lassen, weil *Allerhöchstdieselben grundsätzlich nicht in die Befugnisse der Seekadetten-Annahme-Kommission eingreifen * will. BA-MA, F 3358a/PG 66993 Marine-Kabinett. Einstellungen 1904. 
2. Generalleutnant August v. Madkensen (Danzig/Langfuhr) an den Kontreadmiral und Chef des Marine-Kabinetts v. Müller, 5. 2. 1907.

$B A-M A, F$ 3356/PG 66977. Kais. Marine-Kabinett. Bestimmungen über Einstellungen. gen. $B d 1$.

Euer Hochwohlgeboren beehre ich mich, der Aufforderung vom 3. d. Mts. entsprechend und unter Rüdsreichung sämtlicher Anlagen ganz ergebenst zu berichten, dass die von Gefängniß-Inspektor Dworack in seinem Immediatgesuch 79 über sein Haus gemachten Angaben zutreffend sind. Der Umgang seiner etwa 22-jährigen Tochter mit der erwachsenen Tochter des Generalleutnants v. Seydlitz ${ }^{80}$ ist thatsächlich sogar ein viel näherer, als Inspektor Dworadk in seiner sehr sympathisch berührenden zurüdkhaltenden Würde angiebt. Fräulein Dworack besucht nicht nur die Fräulein v.Seydlizz in deren Elternhause, sondern erhält auch deren Besuch in ihrem Elternhaus und Herr und Frau v. Seydlitz urtheilen über diesen Verkehr durchaus zugunsten der Schwester und des Elternhauses des Walter Dworads.

Jedenfalls ist dieser unter häuslichen Eindrüdsen und Vorbildern aufgewachsen, die neben grösster Anspruchslosigkeit Takt- und Pflichtgefühl in ihm haben wirken müssen. Id würde daher kein Bedenken tragen, den jungen Mann einem der nicht in Danzig garnisonirenden Regimenter der mir unterstellten Division zur Einstellung zu empfehlen. Die Einstellung in einen Danziger Truppentheil würde ich nicht für erwünscht halten im Interesse des jungen Mannes selbst, dem, nicht Eltern und Geschwister, wohl aber entferntere Verwandte am Ort oder in der Nähe vielleicht gesellschaftliche Verlegenheiten bereiten könnten. Die Infanterie-Regimenter in Dtsch. Eylau und Osterode haben sich schon zur Annahme von Fahnenjunkern entschliessen müssen, die ihrer Erziehung und Bildung - wihrer Kinderstube* nach weniger Garantien boten, als Walter Dworads und sein Vaterhaus.

v. Madrensen

Generalleutnant, General-Adjutant Sr. Maj. des Kaisers und Königs, Kommandeur der 36. Division

3. Kontreadmiral und Chef des Marine-Kabinetts v. Müller an den Admiral und Inspekteur des Bildungswesens der Marine v. Arnim, 16. 2. 1907.

$B A-M A, F$ 3356/PG 66977. Kais. Marine-Kabinett. Bestimmungen über Einstellungen. Bd. 1 .

Seine Majestät der Kaiser und König haben auf den Vortrag des Schreibens der (it) vom 1. Februar d. J. ${ }^{\text {B1 }}$ betreffend Ergänzung des Seeoffizierkorps aus Berufsstellungen, welche aus dem Unteroffizierstande hervorgegangen sind (Feuerwerksoffiziere etc.) oder in der Beamtenhierarchie eine entsprechende Stellung einnehmen, nachstehende Allerhöchste Willensäusserung gegeben:

So wünschenswerth es ist, dass das Seeoffizierkorps zahlreichen Zuwachs aus den höchsten Kreisen der Bevölkerung erhält, in denen strenge Ehrbegriffe und gute Formen festgewurzelte Tradition bilden, so nothwendig ist es aus allgemein socialen Gründen unserer Zeit, dass die Offizierslaufbahn einem jungen Mann, der sonst alle Bedingungen erfüllt, nicht deshalb verschlossen sein soll, weil sein Vater sich aus dem Unteroffizierstande emporgearbeitet hat zu den Lebensbedingungen, die ihm gestattet haben, dem Sohne eine höhere Schulbildung zu geben und ihm die Mittel für die Offizierscarriere sicher zu stellen. In diesem hoch anzuerkennenden Emporarbeiten liegt im Gegentheil eine gewisse Gewähr für die allgemeine Tüdhtigkeit der Familie, die wohl erwarten lässt, dass der Sohn aus einer solchen Familie es verstehen werde, die Mängel seiner gesellschaftlichen Erziehung mit Hülfe der zu fordernden Unterstützung durch seine Vorgesetzten bald auszugleichen. 
In der Armee ist es angängig, dass Söhne von Feuerwerksoffizieren etc. die Offizierlaufbahn einschlagen; auch können die Söhne dieser Offiziere im Königlichen Kadettenkorps in Stellen mit vermindertem Erziehungsbeitrag oder in Freistellen Aufnahme finden, falls ihre Väter eine aktive Dienstzeit von 25 Jahren erreicht haben.

Eine Gefahr, dass zu viele Seekadetten aus solchen Kreisen in die Marine eintreten werden, ist schon dadurch ausgeschlossen, dass es den Vätern nur selten möglich sein wird, die erforderlichen Geldmittel zu beschaffen ${ }^{22}$.

Seine Majestät wünschen deshalb, dass die Seekadetten-Annahme-Kommission einen Anwärter nicht lediglich aus dem Grunde zurüdkweist, dass sein Vater aus dem Unter-

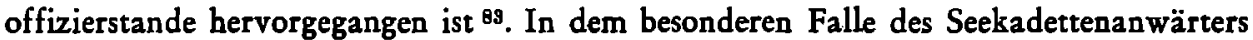
Dworadk befehlen Seine Majestät dass er zu der allgemeinen Konkurrenz bei der Einstellung in diesem Frühjahr einzuberufen ist ${ }^{84}$.

Seine Majestät haben ferner in Bezug auf das von der Kaiserlichen Inspektion angezogene Kabinettsschreiben vom 2. Januar $1904^{85}$ Allerhöchst sich dahin geäussert, dass Seine Majestät Sich durchaus vorbehalten, in besonderen Fällen in die Entscheidung der Seekadetten-Annahme-Kommission einzugreifen - sonst habe ja die Einforderung eines Berichtes der Inspektion des Bildungswesens keinen Sinn - und dass um Missverständnissen nach dieser Richtung hin vorzubeugen, die vorgedruckte Form der Ablehnung von Immediatgesuchen wie in der Anlage zu ändern sei.

gez. v. Müller

4. Kontreadmiral und Chef des Marine-Kabinêtts v. Müller an den Admiral und Staatssekretär des Reichs-Marine-Amts Alfred v. Tirpitz, 16. 5. 1907.

BA-MA, F 6081. Reidss-Marine-Amt. Allgemeines Marinedepartement. IX. 4. 1. - 4. Ergänzung der Seeoffiziere. Heft 7.

Euer Excellenz beehre ich mich beifolgend den Entwurf zu einer Kabinets-Ordre, betreffend die Vorpatentirung der als Abiturienten eingetretenen Seeoffiziere zur Begutachtung vorzulegen.

Ich bin mir nicht zweifelhaft, dass diese oder eine ähnliche Ordre in Seeoffizierkorps mit sehr gemischten Gefühlen entgegengenommen werden wird, nicht immer aus sachlichen Gründen, sondern vielfach deshalb, weil die Seeoffiziere selbst meistens ihre für die Marine bestimmten Söhne nicht erst das Abiturientenexamen machen lassen, sondern den bequemen und billigeren Weg des Eintritts mit Primareife vorziehen ${ }^{80}$. Anderseits bin ich persönlich davon durchdrungen, dass der jetzige Zustand ein unmöglicher ist. Eine Hebung der allgemeinen Bildung des Seeoffizierkorps ist aus rein beruflichen wie aus Standesrüdksichten - hier kommt z. B. aud das Abheben von dem MaschinenIngenieurberuf in Frage - nothwendig. Wird das anerkannt, so ist es auf die Dauer unhaltbar, ja unmoralisch, damit zu rechnen, dass eine sich steigernde Anzahl Offiziersaspiranten jedes Jahr darauf hineinfällt, unter Verlieren von 2 Jahren Anciennität als Abiturienten einzutreten. Thatsächlich hat ja auch in den letzten 3 Jahren eine Steigerung der Prozentzahl Abiturienten nicht stattgefunden ${ }^{87}$.

S. Anm. 70.

вs „Ebenso schob er [Wilhelm II.] Bedenken hinsichtlich der Zulassung von Söhnen von Subalternbeamten oder Feldwebelleutnants zur Offizierslaufbahn glatt zur Seite. Er sagte in einem solchen strittigen Falle: 'Wir können nicht lauter Montmorencys im Seeoffizierkorps haben،. " (Bezieht sich auf Admiral Henri II. de Montmorency). Müller, S. 203.

84 Am 5. 5. 1908 benahrichtigte die Inspektion des Bildungswesens Admiral v. Müller, daß Dworak »ein verdrossener Charakter « sei und ein äußerst schlechter Qualifikationsbericht vorliege: "Sein Auftreten läßt den nötigen Schliff, die exakte Schulbildung und den richtigen Takt vermissen.* Fünf Tage später teilte Müller Dworadss Entlassung zur Reserve Madkensen mit. BA-MA, F 3548/PG 68041 Marine-Kabinett. Fähnrichs zur See und Seekadetten, Bd 4.

85 S. Dok. 1 u. Anm. 78.

86 Reichs-Marine-Amt Randbemerkung (von unbekannter Hand): »das ist der teuere Weg! Schulgeld zahlen ist billiger!^

87 Reichs-Marine-Amt Randbemerkung: »die absolute Zahl steigt noch jetzt jährlich!๔ Vgl. S. $89,92$. 
Eine besondere Berüdssichtigung hat in dem Ordreentwurf die Behandlung von NichtAbiturienten, welche das Offiziersexamen mit Kaisers Belobigung gemacht haben, gefunden. Es ist eine Concession an ganz besonders begabte Nicht-Abiturienten.

In demselben Sinne soll die Schlussbestimmung der Ordre wirken, welche allen, höchste Leistungen versprechenden Offizieren unabhängig von der Art der Einstellung bevorzugte Beförderung in Aussicht stellt und damit überhaupt das "Springen* im Seeoffizierkorps einleitet. Die betreffende Bestimmung ist absichtlich sehr dehnbar gehalten. Sie lässt sowohl den Zeitpunkt wie das Mass des Springens offen und soll zunächst nur das Seeoffizierkorps an den Gedanken des Springens gewöhnen ${ }^{\text {89. }}$

Ich nehme an, dass nach Erlass dieser Ordre die Zahl der Abiturienten unter den Seekadetten schnell zunehmen wird, so dass in einigen Jahren das Abiturientenzeugnis als allgemeine Eintrittsbedingung festgesetzt werden könnte ${ }^{80}$.

Die von Korvetten-Kapitän Fuchs geliefenten Vorarbeiten für die vorliegende Frage gestatte ich mir mit der Bitte um gelegentliche Rüdkgabe beizufügen.

Zum Schluss möchte ich noch gehorsamst anheimstellen, die Ordre von Euer Excellenz aus Seiner Majestät vorzulegen, da sie doch wesentlich in die von Euer Excellenz ressortirende Frage der Ergänzung des Seeoffizierkorps einschneidet ${ }^{80}$.

v. Müller

5. Allerhöchste Kabinetts-Ordre Kaiser Wilhelms II. an den Reichskanzler (ReichsMarine-Amt), 2. 7. 1907.

$B A-M A, F$ 7154/PG 68786. Reichs-Marine-Amt. Zentralabteilung. Akten betr. Allerböchste Kabinetts-Ordres. 3. Vierteljabr 1907. Heft 97.

Idh will in Anerkennung des hohen Werthes, den eine abgeschlossene Schulbildung für den Seeoffiziersberuf hat, den als Abiturienten eintretenden Seekadetten eine spätere Vorpatentirung als Seeoffizier in Aussicht stellen. Die besonderen Anforderungen des Seeoffizierberufes lassen es nothwendig erscheinen, die Vorpatentirung auch von guten praktischen Leistungen während der Dienstzeit als Leutnant zur See abhängig zu machen und sie ferner so zu legen, daß die Ausbildungszeiten für den jungen Offizier nicht gekürzt werden. Es soll deshalb den günstig beurtheilten, als Abiturienten eingetretenen Leutnants zur See bei der Beförderung zum Oberleutnant zur See durch Vorpatentirung ein Vortheil gewährt werden. Im einzelnen bestimme Ih hierzu: 1. Bei der Eingabe der Fähnrichs zur See zur Beförderung zum Leutnant zur See bezeichnet die Inspektion des Bildungswesens diejenigen als Abiturienten Eingetretenen, welche die Offiziersprüfung mit "gut* bestanden haben und auch sonst hinsidhtlich Charakter und praktischer Leistungen geeignet sind, später vorpatentirt zu werden. Diejenigen Fähnrichs zur See, welche als Nichtabiturienten die Offiziersprüfung mit "vorzüglich* bestanden haben und Mir hierfür zur Ertheilung einer Belobigung in Vorschlag gebracht worden sind, sollen derselben Vergünstigung theilhaftig werden. 2. Drei Monate vor dem vorraussichtlichen Zeitpunkt der Beförderung der Betreffenden zum Oberleutnant zur See ist Mir auf dem Dienstwege ein eingehender Bericht darüber vorzulegen, ob eine Vorpatentirung im Sinne Meiner heutigen Ordre empfohlen werden kann. Ausnahmsweise können auch solche als Abiturienten eingetretenen Leutnants zur See zur Vorpatentirung empfohlen werden, welche erst im Laufe ihrer Leutnantsdienstzeit den Beweis erbracht haben, daß sie ihre höheren Schulkenntnisse als Abiturienten in ihrem Berufe richtig auszunutzen verstehen. 3. Die für Abiturienten in obiger Weise geregelte Vor-

s8 Die Organisatorischen Bestimmungen für das Personal des Soldatenstandes der Kaiserlichen Marine v. 26. 6. 1899 hatten Beförderung allein nach der Anciennität geregelt. H. Blads: Die Grundzüge der Beförderungsordnungen, in: Untersuchungen zur Geschichte des Offizierkorps. Anciennität und Beförderung nach Leistung, Stuttgart 1962, S. 126-127 (= Beiträge zur Militär- und Kriegsgeschichte, hrsg. vom Militärgeschichtlichen Forschungsamt, Bd 4).

80 Der Satz ist am Rande durd einen senkrechten Bleistiftstrich hervorgehoben.

00 Am 25. 6. 1907 schrieb Tirpitz an Müller, daß er die AKO befürworte, aber »bitte ich Ew. Hochwohlgeboren den Immediatvortrag bei Sr. Majestät für mich übernehmen u. die Allerh. Ordre erwirken zu wollen «. BA-MA, F 6081, H. 7. 
patentirung tritt zuerst für den Seekadetten-Jahrgang 1909 in Kraft ${ }^{01}$. Abgesehen von vorstehender Regelung behalte Ich Mir weitere Vorpatentirungen von Seeoffizieren, welche Hervorragendes leisten, in Zukunft vor. Sie haben Vorstehendes der Marine bekannt zu machen.

Kiel, an Bord Meiner Yacht "Hohenzollern«, den 2. Juli $1907 . \quad$ Wilhelm I. R.

6. Admiral und Chef der Hochseeflotte Heinrich Prinz v. Preußen an den Vizeadmiral und Chef des Marine-Kabinetts v. Müller, 17. 3. 1908.

$B A-M A, F$ 6082. Reichs-Marine-Amt. Allgemeines Marinedepartement. Ergänzung der Seeoffiziere. Heft 8. Persönliches!

Gelegentlich der von Seiner Majestät befohlenen Bekanntgabe des Schreibens des Marinekabinetts vom 18. Januar d. Js. habe ich die Verbandsführer ${ }^{22}$ beauftragt, Erhebung darüber anzustellen, wie sich die ihnen unterstellten Offizierkorps den jetzt eingetretenen Mangel an Offiziersersatz erklären und eine Besserung dessen für möglich halten.

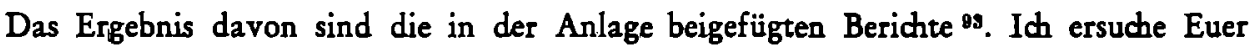
Exzellenz ergebenst, diese Anlagen nach Einsichtnahme dem Staatssekretär des ReichsMarine-Amts zu übersenden, der Absdurift dieses Schreibens erhalten.

Id bemerke zu den Berichten kurz folgendes:

Die Mehrzahl von ihnen führt den augenblicklichen Mangel im Offizierersatz vorzugsweise auf die A.K.O. vom 2. Juli 1907 zurück, die eine Vorpatentierung der Abiturienten in Aussicht stellt. Begründet wird dies damit, daß wohl eine große Anzahl von Anwärtern, die eigentlich mit dem Primanerzeugnis haben eintreten wollen, sich nunmehr veranlaßt gesehen haben, das Abiturientenexamen zu machen. Die Erklärung ist zweifellos berechtigt, bewiesen wird sie erst dadurch werden, daß vom Herbst $1909 \mathrm{ab}$ die Zahl

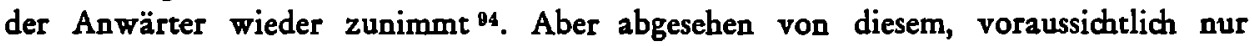
vorübergehenden Einfluß wird die Bevorzugung der Abiturienten, die in absehbarer Zeit das Bestehen des Abiturientenexamens als Bedingung für den Eintritt zur Folge haben wird, für die Zahl und Qualität unseres Offizierersatzes in mancherlei Hinsicht nicht als vorteilhaft bezeichnet ${ }^{05}$. Ich vermag mich diesem Urteil nur anzuschließen. Zur Begründung dessen gehe ich von der Frage aus, welche Anforderungen an den Ersatz des Seeoffizierkorps zu stellen sind.

Unser Beruf verlangt eine, die exakten Wissenschaften und die neueren Sprachen bevorzugende Schulbildung, die den eintretenden jungen Mann befähigt, den Unterricht auf dem Schulschiff und der Marineschule mit Verständnis zu folgen. Er verlangt ferner einen frischen gesunden Körper und Sinn, der die mannigfachen Anstrengungen und Entbehrungen der Ausbildungszeit mit jugendlicher Begeisterung und Leichtigkeit überwindet, und schließlich einen, zu praktischer Betätigung im Leben herangebildeten Charakter. Ohne in Abrede zu stellen, daß unsere humanistischen Gymnasien, die noch weit aus die Mehrzahl der höheren Lehranstalten sind und auch noch allgemein bevorzugt werden, solche Eigenschaften durchaus entwidkeln können, möchte ich andererseits doch darauf hinweisen, daß ihr Lehrplan diesen Forderungen in mancher Hinsicht nicht entspricht. Er ist vielmehr vorzugsweise auf die rein wissenschaftlichen Berufe, besonders den philologischen und theologischen zugeschnitten, und wird aus diesem Grunde auch aus der Mitte vieler Berufskreise, die im praktischen Leben stehen, wie Ingenieure, Arrzte,

91 Die Ordre sollte erst 1909 in Kraft treten, so daß sich das Seeoffizierkorps allmählich an die Idee des Springens gewöhnen konnte und damit ferner diejenigen jungen Männer, die 1908 die Primareife erreichen würden, sich nicht bei der Marine melden sondern bis zum Abitur in der Schule bleiben sollten, um bei der Vorpatentierung berüdksichtigt werden zu können.

92 Vizeadmiral Henning v. Holtzendorff, Kontreadmiral Ludwig Schröder, Kontreadmiral August v. Heeringen u. Kapitän z. S. Wilhelm Lans.

os Diese Berichte wurden von den Verbandsführern sowie von jedem Linienschiffskommandanten eingereidht.

o4 Vgl. S. 92.

os Die letzten Worte des Satzes sind durch einen wazgerechten Bleistiftstridh hervorgehoben. 
Juristen, Kaufleute usw. als veraltet angegriffen; denn die Kenntnisse, die er ins Leben mitgibt, werden von der Mehrzahl aller Schüler zum großen Teil bald über Bord geworfen, als Ergebnis bleibt nur die erworbene allgemeine Befähigung zu geistiger Arbeit zurüdk. Gewiß ist diese hoch zu bewerten, aber nur dann, wenn sie nicht auf Kosten anderer Eigenschaften, die für den Seeoffizier in erster Linie stehen, erworben worden ist. Es ist eine allgemeine Lebenserfahrung, daß theoretische Kenntnisse und praktisches Können oft weit voneinander entfernt sind. Letzteres ist für einen so ausgesprochen praktischen Beruf, wie den des Seeoffiziers, die Hauptsache. Die wesentlichsten Fähigkeiten, durch die sich der Seeoffizier namentlich im Ernstfalle auszeichnen soll, lernt er durch praktische, ich möchte fast sagen handwerksmäßige Betätigung seines Berufs weit mehr, als durch theoretische Ausbildung und wissenschaftliches Studium. Wenigstens gilt dies für den Durchschnittsoffizier, der sich im Borddienst als Wachoffizier, Artillerie-, Navigationsoffizier und Kommandant und nicht in Ausnahmestellungen bewähren soll, für die besondere Fähigkeiten auf wissenschaftlichem, organisatorischem oder anderem Gebiete gefordert werden. Für solche Posten scheiden sid aus einem großen Offizierkorps genügende Persönlichkeiten aus, wie ein Blidk auf unser jetziges älteres Seeoffizierkorps ohne weiteres beweist, von denen nur ganz vereinzelte das Abiturientenexamen gemacht haben ${ }^{\text {06. }}$.

Es ist meines Erachtens außer Zweifel, daß den eigenartigen Lebensbedingungen und Forderungen des Seeoffizierberufs der Mensch im allgemeinen um so besser gerecht wird, je früher er darin heimisch wird. Deshalb halte ich ein möglichst jugendliches Lebensalter zum Beginn der Marinelaufbahn für durchaus erwünscht ${ }^{87}$. Mir erscheint daher das auch vom B. d. A. ${ }^{98}$ befürwortete englische Erziehungssystem als das beste. Es gibt die Mittel in die Hand, die Schulbildung im Hinblidk auf die Forderungen des späteren Berufs zu leiten, die Charaktere frühzeitig zu erkennen und für die Praxis des Lebens zu formen ${ }^{\circ}$. Ob es in Deutschland allgemein Anklang finden wird, lasse ich dahingestellt. Der Deutsche hält im Gegensatz zum Engländer seine Söhne gern länger in der Familie und bevorzugt die rein wissenschaftliche Ausbildung vor der frühzeitigen Entwidkelung der Persönlichkeit und praktischen Betätigung. Beides hat seine Vor- und Nachteile, ein Mittelweg wäre vielleicht das Beste, indem wir ein Marinekadettenkorps schaffen mit zwei Klassen, die etwa der Unter- und Obersekunda eines Realgymnasiums entsprechen. Das Schlußexamen würde mit dem Fähnrichsexamen der Armee gleich zu bewerten sein, sodaß die Schüler, die sich für die Seeoffizierlaufbahn nicht eignen, zur Armee gehen können. Dem entsprechend müßte es auch den Fähnrichen des Lichterfelder Kadettenkorps nach wie vor ermöglicht bleiben, als Seekadetten in die Marine einzutreten. Wie jetzt das Kadettenkorps in Lichterfelde seinen Zöglingen neben der wissenschaftlichen Ausbildung eine tüchtige infanteristische und allgemeine militärische Vorbildung gibt, so müßten auch die Zöglinge des Marinekadettenkorps durch genügende praktische Beschäftigung mit militärischen, seemännischen und technischen Dingen für ihren Beruf vorbereitet werden.

Wird die Einrichtung einer solchen Vorschule nicht für richtig oder möglich gehalten, so sollte es m. E. bei dem jetzigen Verfahren belassen, $d$. h. es sollten Abiturienten und Primaner gleichzeitig und gleichberechtigt ${ }^{85}$ eingestellt werden. Die jetzt in Aussicht genommene Bevorzugung der Abiturienten bringt die Gefahr mit sich, daß im jüngeren Seeoffizierkorps Zwiespalt und Unzufriedenheit entstehen. Namentlich der junge Seeoffizier schätzt praktische Leistungen höher ein, als theoretische. Sieht er sich gegen einen Kameraden, dem er sich als praktischer Seeoffizier ebenbürtig oder überlegen fühlt, zurüdkgesetzt, weil dieser lediglich auf Grund einer höheren wissenschaftlichen

o8 S. Anm. 29.

07 Reichs-Marine-Amt Randbemerkung: wich auch $\alpha$.

o日 Befehlshaber der Aufklärungsschiffe: Kontreadmiral v. Heeringen.

oy Randbemerkung: "Die Frage liegt auch so: wird der Charakter besser von einem sorgsamen Vater im Elternhaus geformt oder im zukünftigen Marine-Kadettenkorps?* A. J. Marder: From the Dreadnought to Scapa Flow. The Royal Navy in the Fisher Era, 1904-1919, Bd I, London 1961, S. 28 ff., für das Erziehungssystem der Royal Navy. 
Vorbildung auf dem Schulschiff und der Marineschule bessere Leistungen erzielt hat, so fühlt er sich nicht mit Unrecht benachteiligt. Wird die Vorpatentierung behufs schnellerer Beförderung besonders befähigter Offiziere für erforderlich gehalten, so muß sie allen denen offen steben, die als praktische Seeoffiziere das Beste leisten. Tritt dagegen die beabsidhtigte Bevorzugung lediglich der Abiturienten in $\mathrm{Kraft}$, so werden sich bald zwei Klassen von Offizieren bilden, die sich feindlidh gegenüberstehen. Allmählich wird sich dann wahrscheinlich der Nichtabiturient völlig verlieren und es werden nur noch Abiturienten eingestellt werden. Dies scheint mir nicht wünschenswert ${ }^{100}$.

Der erste Nachteil ist der, daß die Zahl der sich Meldenden erheblich sinken wird 101. Denn es fallen nicht nur die aus, die das Abiturientenexamen nicht oder nicht frühzeitig genug machen können, sondern auch mancher junge Mann, der mit 16 Jahren für den Seeoffizierberuf schwärmt, wird, bis er sein Abiturientenexamen gemacht hat, in der Wahl seines Berufs wieder schwankend 102. Einzelne bekommen erst in den oberen Klassen den richtigen Geschmadk für wissenschaftliche Beschäftigung und wählen daher einen rein wissenschaftlichen Beruf. Andere schreckt, wenn sie erst mal 18 bis 19 Jahre geworden sind, die Aussicht auf den ihnen bevorstehenden 31/2jährigen scharfen Schulund Dienstzwang zurüd. Sie vergleichen sich mit ihren Schulkameraden, die in der Armee in 11/2 Jahren Offiziere sind oder als Studenten sich völlig unabhängig dünken. Man behaupte nicht, daß solche, ihrer ursprünglichen Berufswahl wieder abspenstig werdende junge Leute für die Marine nichts taugen. In der Jugend läßt sich der Mensch leicht durch Außerlichkeiten beeinflussen, und ist sich über das, was ihm ein Beruf an inneren Werten bietet, oft völlig unklar. Das wirkliche Interesse kommt erst, wenn er seinen Beruf kennen lernt.

Ein weiteres Bedenken der ausschließlichen Einstellung von Abiturienten ist das, daß das Seeoffizierkorps als Ganzes 2 oder 3 Jahre älter werden wird. Sodann wird auch die Herkunft unseres Ersatzes nicht immer günstig dadurch beeinflußt werden. Denn wenn militärische Veranlagungen und Gaben für uns von Wert sind, so müssen wir den Ersatz vorzugsweise aus den Familien suchen, in denen Vererbung, Rasse und Erziehung diesen Geist erwarten lassen. Das sind in erster Linie die alten, guten Offiziers- und Beamtenfamilien. Ihnen wird infolge von häufigen Versetzungen, oft auch durch beschränkte wirtschaftliche Verhältnisse die Erziehung der Kinder schwer. Sie sind dazu gezwungen, diese frühzeitig aus dem Hause zu geben und sie auf eigene Füße zu stellen. Die Forderung des Abiturientenexamens verlangt erheblich höhere Kosten, die manchen Vater davon abhalten werden, seinen Sohn Seeoffizier werden zu lassen. Auch hat mancher Junge aus diesen Kreisen, der auf der Schule nicht recht fortkommt, und das Abiturientenexamen nicht oder nicht früh genug machen kann, durchaus das Zeug zu einem tüchtigen Seeoffizier und geht uns verloren.

Die Ausschaltung solcher uns erwünschter Elemente zwingt auf andere Kreise überzugehen, deren Söhne vielleicht eine höhere wissenschaftliche Bildung, aber weder die Erziebung noch die sittlichen und sozialen Anschaungen mitbringen, die jene verbürgen ${ }^{103}$. Je mehr wir aber solche Elemente bekommen, desto mehr werden grade die Kreise der Marine fern bleiben, die seit Jahrhunderten die festesten Stïtzen unserer Wehrmacht gewesen sind und in bewußtem Gegensatz zu der in Deutschland oft übertriebenen Bevorzugung wissenschaftlicher, auf das Abstrakte gerichteten Betätigung, die

100 Am 1. 4. 1908 antwortete Müller Prinz Heinrich: *Von einer Bevorzugung der Abiturienten in der Seeoffizierlaufbahn könnte doch nur die Rede sein, wenn sie durch Vorpatentirung mehr gewinnen würden als die zwei Jahre, um weldhe sie später eintreten. Das ist aber gar nicht der Fall. Es ist im Gegenteil nur in Aussicht genommen, sie um ein Jahr vorzupatentiren.* BA-MA, F 6082, H. 8.

101 Randbemerkung: what sich durchaus richtig erwiesen!

102 Randbemerkung: srichtig«.

103 Am 1. 4. 1908 antwortete Müller: Der Zustand, dass das Seeoffizierkorps nicht in erwünschtem Maasse Nachwuchs aus den geeignetsten Gesellschaftsschichten erhält, besteht schon seit Jahrzehnten. Er kann deshalb nidht wohl mit der Abiturientenfrage oder mit der jetzt aufgeworfenen Frage der allgemeinen Berufsfreudigkeit in Verbindung gebrache werden.* BA-MA, F 6082, H. 8. 
Pflege männlicher, soldatischer und mit der Praxis des Lebens rechnender Tugenden hochgehalten haben 104.

Dieser Verlust wäre für das Seeoffizierkorps sehr zu beklagen.

Besonders schwierig ist für den Seeoffizier die Erziehung und Schulbildung seiner Kinder.

Er wird recht häufig versetzt und ist soviel abwesend, daß ihm oft jeder persönliche

Einfluß hierauf fehlt. Grade zur Pflege der im Seeoffizierkorps allmäblich sich bildenden

Tradition und Rasseeigenschaften ist es dringend erwünscht, uns dessen Nachwuchs nach Möglichkeit zu wahren. Die oben genannte Marinevorschule wäre das Beste hierzu. Kommt es nicht zu dieser, so muß unbedingt Fürsorge dafür getroffen werden, daß zwischen bestimmten Schulen in unseren Hauptgarnisonorten, am besten Realgymnasien, ein organischer Zusammenhang geschaffen wird, der die Anforderungen der gleichen Klassen auf gleicher Höhe erhält und den Ubergang von einer Schule zur andern ohne Prüfung und ohne Zurüdkbleiben ermöglicht.

Wie Eure Exzellenz aus meinem beigefügten Schreiben an die Verbände G. 59 A. 1 vom 3. II. d. Js. ersehen werden, habe ich auch Erhebungen darüber anstellen lassen, ob der Mangel an Meldungen vielleicht darauf zurüdkzuführen ist, daß der junge Seeoffizier nicht mehr die in ihm früher eigene Berufsfreudigkeit besitzt und daher aus eigenem Antrieb nicht mehr für sein Korps wirbt. Dabei ist die mir schon früher bewußte Tatsache bestätigt worden, daß sich im jüngeren Seeoffizierkorps zur Zeit eine gewisse Unzufriedenheit geltend macht. Idh bin weit davon entfernt dieser augenbliddlichen Stimmung allzugroßen Wert beizumessen, erkläre sie mir vielmehr in der Hauptsache durch den jedem jungen Menschen innenwohnenden Drang, bei allen Entbehrungen und Enttäuschungen, die ihm sein Beruf bietet - und das tut jeder Beruf - sich in vorschneller Kritik Luft zu machen. Einzelne Punkte möchte ich aber doch hervorheben:

Die herbste Enttäuschung erlebt der mit Begeisterung in die Marine eintretende junge Mann dadurch, daß ihm die erhoffte Gelegenheit, ins Ausland zu kommen und die Welt zu sehen, so selten geboten wird. Die Klage über die geringe Zahl der Auslandskommandos ist ganz allgemein und bedarf der Berüdksichtigung ${ }^{105}$. Der jetzige Zustand, der ja zum Teil durch die wachsende politische Bedeutung unserer Flotte entstanden ist ${ }^{100}$, läßt sich vielleicht allmählich ändern. Zunächst würde es sich wohl empfehlen, auf allen Auslandsschiffen Leutnants zur See in der größtmöglichsten Zahl über den Etat einzuschiffen. Als weiteres Mittel möchte ich vorschlagen, die Reisen der Seekadettenschulschiffe zu verlängern auf eine Dauer von etwa Mitte Mai ${ }^{107}$ bis Ende März, also 10 Monate, und sie dann so einzurichten, daß die Seekadetten auch etwas eigenartiges zu sehen bekommen. Jetzt verlassen die Schulschiffe erst Mitte Juli die heimischen Gewässer und gehen in der Mehrzahl ins Mittelmeer. Die Reisen dorthin bieten den Seekadetten wenig ${ }^{100}$. Der junge, zum ersten Mal ins Ausland kommende Mann will etwas ganz anderes sehen, als das was es zu Hause gibt und dort allgemein bekannt ist. Das Anlaufen so vieler Häfen, in denen sich Feste auf Feste stürzen verwöhnt ihn. Vor allem aber bietet keine Gegend auf der ganzen Welt so viel Gelegenheit zu Verführungen und Verirrungen auf geschlechtlichem Gebiet, wie das Mittelmeer. Auch die allgemeinen Gesundheitsverhältnisse sind dort nicht gut ${ }^{100}$. Man sollte die

104 *Nach Vorstehenden kann wohl kaum aufrecht erhalten werden, dass reine einseitige Betonung der wissenschaftlichen Bildunge vorliegt. Der Vorwurf wird aber erst recht hinfällig, wenn man bedenkt, dass die Kabinets-Ordre vom 2. Juli 1907 nur die Vorpatentirung der Abiturienten vorsieht, welche die Offiziersprüfung mit 'gut bestanden haben und auch sonst binsidblich Charakter und praktisden Leistungen geeignet sind, später vorpatentirt zu werden.* Müller an Prinz Heinrid, 1. 4. 1908. BA-MA, F 6082, H. 8.

105 In seinem Schreiben v. 1. 4. 1908 an Prinz Heinrich pflichtete Müller diesem in dieser Hinsicht vollkommen bei: $*$ In Bezug auf das Wünschenswerthe der Auslandsreisen besteht wohl an keiner Stelle der Marine ein Zweifel.* BA-MA, F 6082, H. 8.

$100 \mathrm{Vgl.} \mathrm{S.} \mathrm{91.} \mathrm{Ferner} \mathrm{Berghahn,} \mathrm{S.} 68$ ff. sowie Kennedy, S. 33-56.

107 Randbemerkung: "Das würde noch verschiedene Vortheile haben. Die Seek. Schulschiffe werden durch die Kieler Woche nicht gestört u. das unsinnige Wetteifern fällt fort. *

108 Randbemerkung: "S. Maj. der Kaiser sind andrer Meinung.e

100 Randbemerkung: *Man kann die Reisen so einreihen, daß die Feste versäumt werden. Mittelmeer bietet außerordentlich viel Anregendes.« 
Schulschiffe weiter weg von der Heimat schidken, nach Ostasien und zurüdk, um Afrika herum, nach Westindien und Südamerika usw. Die dabei entstehenden höheren Indiensthaltungskosten müssen der Wichtigkeit der Sache entsprechend, in Kauf genommen werden. Mit den längeren Seereisen wären auch längere Hafenliegezeiten zu verknüpfen, die den Eigenarten beider gerecht werden und der Ruhe und Gleichmäßigkeit des Dienstbetriebes zu Gute kommen ${ }^{110}$.

Ein weiterer Punkt sind die Klagen der jüngeren Offiziere über die Mängel ihrer gesellschaftlichen Stellung. Sie sind zum Teil in der ungewöhnlich großen Ansammlung von Seeoffizieren in Kiel begründet. Dort spielt der junge Offizier in Vergleich mit seinen Kameraden der Armee, der in allen Garnisonen die Hauptstütze der Geselligkeit ist, fast gar keine Rolle. 4 bis 5 Tage in der Woche ist er in See, Wachverpflichtungen beschränken ihn an den übrigen Tagen. Das Verbot, sich eine Landwohnung zu halten erschwert ihm die Teilnahme an der Geselligkeit noch mehr ${ }^{111}$. Er muß, wenn er nachmittags an Land gegangen ist, zum Umziehen an Bord seines Schiffes zurüdkkehren, das weit draußen liegt, muß nach Beendigung von Gesellschaften oder sportlichen Öbungen einen langen Weg zur Landebrüdke zurüdklegen und dort wohlmöglich noch längere Zeit auf das Routineboot warten 112. Das alles ermuntert nicht dazu, jede Gelegenheit zum geselligen Verkehr auszunutzen. Die von Euer Exzellenz auch für richtig erachtete Maßnahme, wenigstens den Oberleutnants zur See auf ihren, vor dem Kommandanten begründeten Wunsch das Halten einer Landwohnung zu gestatten, verspricht hier einige Abhilfe. Ebenso wird die Verlegung eines Teiles der Hochseeflotte nach Wilhelmshaven günstig wirken. Die Heranziehung der jüngeren Offiziere zur Geselligkeit ist aber ein Gegenstand, der der Beachtung bedarf 119. Gerade die aus guten Kreisen stammenden Herren, die die Annehmlichkeiten und den Segen eines ungezwungenen geselligen Verkehrs kennen, entbehren diesen am meisten.

Einzelne Berichte heben hervor, daß der Mangel des Ersatzes ein Zeichen unserer Zeit ist, in der infolge der langen Friedensperiode ganz allgemein dem Heer und der Flotte nicht mehr die gebührende Wertschätzung zu Teil wird. In mancher Hinsicht mag dies für die Armee zutreffend sein. Es wird sich aber in der Marine, die in ibrer Bedeutung der Nation jetzt erst voll vor Augen getreten ist, sich eines wachsenden Interesses erfreut, und auch schon im Frieden positive Arbeit bietet sowie günstige Beförderungsbedingungen in Aussicht stellt, kaum bemerklich machen, wenn wir den Kreisen aus denen wir unseren Ersatz vorzugsweise erstreben, den Eintritt nicht über Gebühr erschweren 114. Wir dürfen daher m. E. die Anforderungen an wissenschaftliche Vorbildung nicht zu hoch schrauben und müssen jedem jungen Mann, der eintritt, die gleichen Bedingungen für ein bevorzugtes Avancement geben. Bei aller Einschätzung des Wertes einer guten wissenschaftlichen Vorbildung darf $\mathrm{m}$. E. deren Bedeutung für unser Offizierkorps nicht überschätzt werden. In langen Friedensperioden liegt diese Gefahr vor. $\mathrm{Da}$ kommen wissenschaftlich besonders gut gebildete, feder- und wortgewandte 102 Offiziere leichr ins Vordertreffen. Im Kriege beschränkt sich die Arbeit auf rein geistigem Gebiete auf wenige Persönlichkeiten. Sonst geben seemännischer Blidk, praktische Erfahrung, frische

110 Randbemerkung: wrichtig«. "Wenn wir sie nicht bedeutend erhöhen, ganz unmöglich. Wir können schon jetzt nicht das Nothwendigste für die Ausbildung in Artillerie- u. Torpedowie Torpedobootswesen leisten.*

111 In Bezug auf das Verbot der Landwohnung für jüngere Offiziere der Flotte möchte ich daran erinnern, dass die bezügliche Allerhöchste Willensäusserung hervorgerufen ist durch einige sehr ernste Straffälle, aus denen ein völliger Mangel an Kontrolle der Lebensführung der betreffenden jungen Offiziere hervorging. " Müller an Prinz Heinrich, 1. 4. 1908. BA-MA, F 6082, H. 8.

112 Randbemerkung: * Wozu dann noch die Verpflichtung des Wachhab. zur Zeit für d. Theater? *

11 Randbemerkung: Wenn die Marineschule nach Mürwik verlegt [wird], wird dem Seeoff.aspiranten der gesellschaftliche Umgang noch mehr erschwert [!].*

114 Am 1. 4. 1908 schrieb Müller an Prinz Heinridh: „Die hohe Bewerthung des Abiturientenexamens ist gerade für die Kreise charakteristisch, aus denen wir gern unseren Offizierersatz haben möchten. Die bisherige gänzliche Negirung des Werthes des Abiturientenexamens in der Marine hat uns manchen erwünschten Jüngling aus Familien fern gehalten, in denen das Abiturientenexamen als Bildungsstab Tradition ist.* BA-MA, F 6082, H. 8. 
Initiative und gesunde Nerven den Ausschlag. Diese Eigenschaften in unserem Seeoffizierkorps zu züchten, ist die vornehmste Aufgabe unseres Erziehungssystems ${ }^{115}$. gez. Heinrich Prinz von Preußen

7. Admiral und Staatssekretär des Reichs-Marine-Amts v. Tirpitz an den Vizeadmiral und Chef des Marine-Kabinetts v. Müller, 8. 4. 1909.

$B A-M A, F$ 6082. Reichs-Marine-Amt. Allgemeines Marinedepartement. Ergänzung der Seeoffiziere. Heft 8 (Sofortl)

Wenn in diesem Jahre auch eine kleine Steigerung der Anmeldungen zur Seeoffizierlaufbahn erfreulicher Weise zu verzeichnen ist, so scheint es mir doch nicht unbedenklich, daraufhin Maßnahmen im Sinne des Schreibens Ew. Exz. vom 18. März ins Auge zu fassen. Der von der Inspektion des Bildungswesens zu erwartende Bericht 110 wird zunächst Aufschluß darüber geben, ob die Einstellung an Abiturienten in gleichem Maße zugenommen hat, wie die Anmeldung, d.h. ob die sich meldende Abiturienten auch in sozialer und in körperlicher Hinsicht den an den Seeoffizierersatz zu stellenden Anforderungen genügen.

Mit Ew. Exz. bin ich der Ansicht, daß der Seeoffizier im Durchschnitt nicht eine geringere Schulbildung besitzen soll, wie andere Offizier- und Beamtenkategorien in der Marine. Andererseits aber würde ich es nicht für richtig halten, solche jungen Leute vom Seeoffizierberuf grundsätzlich auszuschließen, die durch häufige Versetzung ihrer Väter (Söhne von Offizieren und höheren Staatsbeamten) oder durch Erziehung außerhalb des Elternhauses (Söhne von Gutsbesitzern) die Schule unter ungünstigen Bedingungen besuchen mußten und in Folge dieser Verbältnisse, nicht aus Mangel an Begabung, die Abiturientenreife zu spät erlangt haben, um noch für die Seeoffizier-Laufbahn in Betracht zu kommen. Damit würden uns gerade diejenigen Elemente entgehen, die wegen ihrer Herkunft und Erziehung als die geeignetsten anzusprechen sind. Auch würde die Forderung höherer Schulbildung eine Erschwerung der Eintrittsbedingungen für die Zöglinge des Kadettenkorps bedingen, wogegen Bedenken vorliegen.

Schließlich ist es nicht von der Hand zu weisen, daß der Eintritt in die Laufbahn in möglichst jungen Jahren nicht geringe Vorteile bietet, zumal in nicht ferner Zeit eine wesentliche Verschlechterung des Avancements Platz gegriffen haben wird. Soweit mir bekannt, werden im Seeoffizierkorps diese Vorteile besonders hoch bewertet.

Sollte es sich aber in den nächsten Jahren herausstellen, daß der Ersatz an Abiturienten in Bezug auf Herkunft, körperliche Brauchbarkeit und Lebensalter allen Anforderungen entspricht, so wird sich die Frage voraussichtlich von selbst in der Weise regeln, daß Primaner und Fähnriche nur noch ausnahmsweise zur Einstellung kommen. Ich möchte einer solchen organischen Entwicklung den Vorzug geben.

Von der Einstellung mit der Reife für Oberprima verspreche ich mir keine nennenswerthen Vortheile, weil m. E. durch den Besuch der Unterprima weder eine abgeschlossene Schulbildung noch ein gleichmäßiger Ausgangspunkt für die fachmännische Ausbildung erreicht wird.

Aus diesem Grunde, und weil sich die Zahl der sich meldenden Abiturienten ohnehin vermehrt hat, scheint mir zur Zeit ein genügender Anlaß zur Anderung der bestehenden Bestimmungen nicht gegeben zu sein.

T[irpitz]

115 Randbemerkung: sstimmt*.

116 Es handelt sich um den Bericht des Inspekteurs des Bildungswesens der Marine (Vizeadmiral Bordkenhagen) an Tirpitz am 23. 6. 1909: *Man kann wohl mit Recht sagen, daß der beschrittene Weg zu dem gewünschten Ziele führte, ein Offizierkorps zu schaffen, das im Stande ist, den Anforderungen zu entsprechen, die der schnelle Fortschritt auf allen Gebieten der Kriegswissenschaften in einer Marine in geistiger Beziehung stellt. * Bordkenhagen stellte fest, daß die Abiturienten in theoretischer, wie in praktischer Leistung vor den Primanern und Fähnrichen rangierten; im Seeoffizierexamen von 1909 erhielten die Abiturienten 62,5, die Primaner 43,6 u. die Fähnriche 37,0 Durchschnittspunkte. Trotzdem befürwortete Bordsenhagen den Eintritt von mehr Primanern: "Sie übertragen in unseren Offizierersatz die gute Tradition der Armee und in sozialer Beziehung stammen sie zumeist aus den Kreisen, aus denen wir in unser Offizierkorps möglichst viele Anwärter hineinnehmen müssen«, m. a. W. -Söhne von Offizieren, höheren Beamten und Gutzbesitzern«. MA-MA, F 6082, H. 8. 
8. Vizeadmiral und Chef des Marine-Kabinetts v. Müller an den Admiral und Staatssekretär des Reichs-Marine-Amts v. Tirpitz, 4. 5. 1909.

BA-MA, Nadhlaß v. Tirpitz, N 253, Bd 17. Akten betr. Briefe 1909-1913.

Eure Exzellenz bitte ich noch einmal auf die Abiturientenfrage zurüdkzukommen zu dürfen, die mir in dem amtlichen Schreiben des R.M.A. vom 8. April d. Jr., AId $2522{ }^{117}$, nicht in dem Geiste behandelt zu sein scheint, wie Eure Exzellenz mir persönlich immer gegenüber gestanden haben und wie sie meiner Ansicht nach in der Praxis auch ganz gut behandelt werden kann. Eure Exzellenz werden mich nicht mißverstehen, wenn ich hier $z$ wischen amtlicher und persönlicher Auffassung unterscheide, kommt es doch in allen Dienststellungen leider nur zu oft vor, daß die amtliche Stellungnahme sich nicht voll mit der persönlichen Ansicht dedkt, sondern einen Kompromiß zwischen dem Erwünschten und der Rüdrsichtnahme auf Vorhandenes darstellt.

Mein Vorschlag ging dahin, man solle die Minimalschulbildung für die Seeoffizierslaufbahn von der Reife für Unterprima auf die Reife für Oberprima hinaufsetzen. Hiermit werde dank der möglichen späteren Vorpatentierung der Abiturienten um $1 \mathrm{Jahr}$ eine unwiederbringliche Benachtheiligung der Abiturienten gegenüber den Primanern, wie sie jetzt noch vorhanden ist, vermieden. Es werde ferner damit der Ubelstand beseitigt, daß der Seeoffizierersatz mit geringerer Schulbildung angenommen wird als der Zahlmeisterersatz. Die Möglichkeit des Hinaufsetzens der Eintrittsbedingungen sei durch die reichliche Anmeldung zur Seeoffizierslaufbahn gegeben.

Das Antwortschreiben des R.M.A. stellt sich demgegenüber auf den Standpunkt, es genüge, wenn der Durchschnitt des Seeoffizierersatzes mit höherer Shulbildung eintrete als der Ersatz der Zahlmeister u. Ingenieurlaufbahn. Das ist mir ganz unverständlich. Es kommt meiner Ansicht nach durchaus darauf an, daß das Prinzip mindestens gleich hoher Schulbildung für das Seeoffiziercorps durchgeführt wird. Das ist eine widhtige Forderung für die allgemeine dienstlidhe u. soziale Stellung des Seeoffiziercorps. Bei der Zulassung zur Zahlmeisterlaufbahn genügt es, wenn der Durdsschnitt nur die erwünschte Schulbildung hat, denn hier ist nicht, wie bei der Seeoffizierlaufbahn, ein Prinzip zu wahren.

Das R.M.A. führt gegen die Forderung der Reife für Oberprima aus, es sei die Erreihung der Oberprima kein organischer Schulabschnitt. Es muß auffallen, daß diese Oberlegung nicht angestellt worden ist, als für die Zahlmeistercarriere die Reife für Oberprima als Eintrittsbedingung festgelegt wurde. Aber eine derartige Uberlegung würde ebenso gut auch die Festsetzung der Reife für Unterprima als Eintrittsbedingung ausschließen, denn der organische Schulabschnitt liegt nicht beim Ubergang von Obersecunda nach Prima, sondern beim Ubergang nach Obersecunda (Einjähriges Zeugniß). Hierüber ist offenbar der Dezernent im R.M.A. nicht unterrichtet gewesen. Idh habe auf diesen Punkt in meiner vor etwa 3 Jahren in der Mar. Rundschau veröffentlichten Betrachtung über "Seeoffizierlaufbahn und Schulbildung" aufmerksam gemacht ${ }^{118 .}$ Es führt dann das R.M.A. gegen mich ins Gefecht, man würde durch Erhöhung der wissenschaftlichen Eintrittsbedingungen die Offiziersöhne, die durch häufigen Schulwechsel leicht zurüdkkommen, von der Karriere ausschließen. Ich habe mir darauf hier die diesjährige Einstellung von Offizierssöhnen angesehen und gefunden, daß unter 40

117 Dok. 7.

118 Von einem Seeoffizier: Schulbildung und Seeoffizierlaufbahn, in: Marine-Rundschau, 16 (1905), S. 829-835, wo Müller forderte, $\rtimes$ daß von den Seeoffizieraspiranten das Abiturientenzeugnis als Aufnahmebedingung in den Beruf gefordert werde. Die in der Primareife ausgedrüdste Schulbildung, die jetzt als Bedingung für die Einstellung als Seekadett festgesetzt ist, genügt schwerlich in einer Zeit, in der die preußische Armee eifrig dahin wirkt, daß der Offizierersatz das Abiturientenzeugnis erworben haben soll. « Müller gab zu, daß das Erreichen des Abiturientenzeugnisses mgerade bei dem erwünschten Seeoffizierersatz, den Söhnen aus Offiziersfamilien, verhältnismäßig selten sei. Trotzdem beharrte Müller auf seinem Standpunkt: Aber diese höhere Shulbildung ist doch ein so großer, die soziale Stellung des Offizierstandes sehr wesentlich mitbestimmender Faktor, daß sie die Regel für den Offiziernachwuchs bilden sollte.* 
Offizierssöhnen nur 15 nicht das Abiturientenexamen gemacht haben ${ }^{119}$. Hätten diese 15 mit ihrem Eintritt in die Marine gewartet, bis sie die Reife für Oberprima erlangt haben, so würden darunter nur 6 das Durchschnittsalter der eingestellten Abiturienten (19 Jahre) überschritten haben und auch unter diesen 6 würde keiner über 20 Jahre alt sein, wie dies 14 von 155 Abiturienten sind. Diese Zahlen lassen deutlich erkennen, wie unzutreffend die Bedenken des R.M.A. gegen meinen Vorschlag sind. Es würde einfach kein einziger der angenommenen Offizierssöhne von der Seeoff. Laufbahn ausgeschlossen sein, wenn die Eintrittsbedingung auf Reife für Oberprima hinaufgesetzt worden wäre. Wohl aber wäre eine sehr viel gleichmäßigere Vorbildung des gesamten Jahrganges vorhanden, welche die fachmännische Ausbildung wesentlich erleichtern würde. Auch könnte die allgemeine Behandlung der Seekadetten eine gehobenere sein, wenn nicht, wie jetzt, Altersunterschiede $\mathrm{zwischen} 164 / 12$ und $20 \% / 12$ vorhanden wären.

Es ist vom R.M.A. allgemein die großmöglichste Jugend des Seeoffiziercorps betont worden. Nun, das Durchschnittsalter des als Seekadett eintretenden Abiturienten beträgt in diesem Jahre 19, das der Primaner 181/4 Jahr[e] ${ }^{120}$. Für das höhere Alter von $1 / 4$ Jahren habe ich also 2 Jahre - und zwei sehr ausschlaggebende Jahre - allgemeiner Bildung gewonnen. Das ist doch sicher ein sehr vortheilhaftes Geschäft für die Marine, es sei denn daß man sich, wie es allerdings noch manche ältere Seeoffiziere fertig bekommen, auf den Standpunkt stellt, daß der Abiturient schon ein überbildeter Mensch ist, der den freien Blick für die Praxis verloren hat. Spielt das Lebensalter wirklich eine so große Rolle, so soll man entschlossen zu dem früheren Einstellungsverfahren (Obersecunda Reife und strenge Altersbeschränkung) zurüdkgehen und in den Kauf nehmen, $\mathrm{da} B$ bei dem allgemeinen Bildungsniveau unserer Ing. die Seeoffizierslaufbahn einen subalteren Anstrich bekommt 121.

Es ist mir schließlich auch vorgahalten, mit dem späteren langsameren Avancement wäre eine Gefahr der Überalterung des Seeoffiziercorps verbunden, die es künftighin besonders wichtig mache, daß die Seekadetten jung eintreten. Id möchte dagegen geltend machen, daß bei verringerter Einstellung von Seekadetten die Auswahl wächst und $\mathrm{da} B$ man diese Auswahl unter anderem auch ausnutzen wird, zu alte Anwärter abzulehnen. Dann haben wir aber auch noch den Trumpf des bevorzugten Avancements in der Hand (das Springen), so daß die verschwindendgeringe Steigerung des Durchschnittsalters durch Hinaufsetzen der Eintrittsbedingung von Unter- auf Oberprimareife in keiner Weise eine Gefahr bedeutet.

Eure Exzellenz sind mir eine Zeuge dafür, daß ich von jeher für die Abiturienten als den zwedkmäßigsten Seeoffizierersatz eingetreten bin. Wenn ich es jetzt mit besonderem Eifer thue, so spricht dabei außer der immer mehr gesteigerten Uberzeugung von der Richtigkeit meines Standpunktes ein persönliches Motiv mit, indem ich mich in die Seele meines Sohnes hineinversetze, der vielleicht in Jahr und Tag vor die Frage gestellt wird, ob er in die Marine eintreten will oder kann. Da hat er nun Kameraden im Kadettenkorps, die, weit unter ihm in der Klasse sitzend, zu unbegabt oder zu indolent sind, um in die Prima übernommen werden zu können, die aber mit der Prima Reife, bzw. dem Fähnrichsexamen in die Marine eintreten werden. Diese Kameraden werden nicht nur 2 Jahre früher Offizier, sondern sie bleiben auch später, selbst wenn durch das Springen zum Oberleutnant mein Sohn $1 \mathrm{Jahr}$ wieder einholen sollte, für immer in der

110 Randbemerkung: Das ist doch ein sehr hoher Prozentsatz. Unter Off. Söhnen sind 62,5\% Abit. gegenüber 77,5\% v. Ganzen. *

120 Randbemerkung: "Das ist zufällig. Bei der ungleichen Zahl auf beiden Seiten ist ein Vergleich überhaupt nicht möglich.※

121 Vgl. den Bericht des Inspekteurs des Bildungswesens der Marine (Admiral Coerper) v. 24.1.1912 an das Reichs-Marine-Amt. BA-MA, F 3356/PG 66977. Coerper warnte Tirpitz, daß bereits vier Brüder von Marineingenieuren oder Ingenieur-Aspiranten sich zur Seeoffizierlaufbahn gemeldet hatten: $\bowtie$ Es ist unausbleiblich, daß eine solche Gleichstellung des Ersatzes - auch wenn sie auf wenige Fälle beschränkt bleibt - bei den Marineingenieuren das Gefühl der Unzufriedenheit mit ihrer militärischen und gesellschaftlichen Stellung und mir ihren Beförderungsverhältnissen vermehren, dem Agitationsbedürfnis neuen Stoff zuführen wird und unerquidkliche Verhältnisse in den Offiziermessen schafft.e 
Anciennität höher. Das ist doch geradezu eine Prämie auf minderwerthige Schulleistungen und umgekehrt ein so schlechter, direkt herabwürdigender Start für einen tüchtigen Schüler, der das Abiturium machen soll - und in sehr vielen Familien ist das Tradition - daß sicher mancher sehr erwünschter Ersatz dadurch von der Seeoffiziercarriere abgeschredkt wird.

Ich kann nur noch einmal wiederholen, daß ich die Seeoffiziere nicht verstehe, welche sidh dagegen sträuben, den Abiturienten wenigstens so weit Gerechtigkeit widerfahren zu lassen, als es möglich ist, ohne diejenigen jungen Leute aus guten Familien ganz auszuschließen, welche in Folge besonderer Verhältnisse außer stand sind, das Abiturium einigermaßen in normalem Alter zu bestehen. Die Armee würde glüdklich sein, wenn sie in der Lage wäre, ihre Eintrittsbedingungen in die Höhe zu setzen und sich ganz aus Abiturienten zu rekrutiren. Wir könnten dem wenigstens sehr nahe kommen. Es wäre ein schwerer Fehler, wenn wir diese Lage nicht ausnützten.

Idh hoffe, daß Eure Exzellenz noch einmal mit Admiral Bordkenhagen hierüber sprechen, dem ja die reichsten Erfahrungen auf diesem Gebiete zur Verfügung stehen 110. Vielleicht lassen Eure Exzellenz ihn sich zu meinen heutigen Ausführungen äußern.

v. Müller

9. Vizeadmiral und Inspekteur des Bildungswesens der Marine Hans v. Dambrowski an den Großadmiral und Staatssekretär des Reichs-Marine-Amts v. Tirpitz, 7. 4. 1913. $B A-M A, F$ 6082. Reichs-Marine-Amt. Allgemeines Marinedepartement. IX. 4. 1. 4. Ergänzung der Seeoffiziere. Heft 10.

Die Marine tut gut daran, sich jederzeit eine große Zahl von nicht zu alten Abiturienten zu sichern. Wägt man ihre Vorzüge und Mängel gegeneinander ab, so kann es nicht zweifelhaft sein, daß die Abiturienten dasjenige Offiziermaterial herzugeben berufen sind, von dem eine in der Entwidkelung schnell fortschreitende, auf der Höhe der Leistungen zu erhaltende moderne Marine, die ohne gediegene Geistesarbeit und Arbeitsfähigkeit ihres Seeoffizierkorps einfach nicht bestehen könnte, großen Nutzen haben wind, sofern diese Abiturienten neben ihrer wissenschaftlichen Überlegenheit auch den sonstigen an spätere Offiziere zu stellenden Forderungen genïgen. Jungen, geistig regen, für den Beruf begeisterungsfähigen, aus erwünschten Kreisen kommenden Abiturienten würde ich stets den Vorzug geben, ihre Mängel gern in Kauf nehmen. Die Erfahrung jedes neuen Jahres läßt jedoch keinen $Z$ weifel daran, daß die Zahl soldher wünschenswerten Abiturienten klein ist 122. Gegenüber den hier geschilderten jungen Leuten finden wir unter den Anmeldungen der letzten Jahre zahlreiche Abiturienten, die für unsern Zwedk zu alt, nach Herkunft, persönlicher Beschaffenheit, Erziehung wenig geeignet, häufig vollkommen ungeeignet sind. Auf gewisse Eigenschaften unseres Nachwuchses können wir aber, einfach um unser Korps auf der Höhe der Brauchbarkeir und Wertschätzung zu erhalten, nicht verzichten und aus dem Grunde sind wir gezwungen, uns, soweit die Abiturienten diese unerläßlichen Eigenschaften nicht besizzen, unsern Bedarf durch Heranziehung geeigneter Primaner zu dedken. Ein Offizierkorps, dessen Bewertung darin gipfelt, daß es in allen seinen Gliedern über die abgeschlossene Schulbildung verfügt, dieses aber auf Kosten der ritterlichen und traditionellen Eigenschaften des deutschen Offizierstandes, ist für die Marine nicht erstrebenswert. Es wird nach innen und nach außen versagen. In der Armee ist $e s$ auch nicht der Fall, ohne daß es ihr schadet. Dasjenige Offizierkorps, in welchem neben durchschnittlich guter Geistesbildung hergebrachter Schneid, tadellose Sitte und häusliche Erziehung heimisch sind, wird sich letzten Endes am besten durchsetzen. Ist das nur dadurch zu erreichen, daß man zum Teil auf das Reifezeugnis verzichtet, so tritt dieser Mangel jenem Hauptbedürfnis gegenüber in den Hintergrund.

122 Randbemerkung von Vizeadmiral Günther v. Krosigk: „Qualitativ wünschenswerthe Abiturienten nicht in geniigender Zahl vorhanden.* *Also*. (Krosigk war Direktor des Allgemeinen Marinedepartements des Reichs-Marine-Amts). 
Es wird uns also, wenn wir uns auch für die Zukunft eines wirklichen deutschen Offizierkorps in der Marine erfreuen und es nicht erleben wollen, daß hergebrachter Offiziergeist und Sitte bei uns mehr und mehr verschwinden, nur der Weg bleiben, gute Primaner in größerer Zahl neben den Abiturienten heranzuziehen ${ }^{129}$. Vergegenwärtigen wir uns die zahlreichen und unbestreitbaren Vorzüge der Primaner und prüfen wir vorurteilsfrei das, was uns diese und was jene bringen, so wird man zugeben müssen, daß gute Primaner bis zu einer gewissen Zahl uns geradezu erwünscht sein müssen. Eine angemessene Mischung ist der Eigenart der Marine, wie mir scheint, nur zuträglich: junge frische Elemente neben den gereifteren vorgeschrittenen Abiturienten 124. Von keinem Offizierkorps so sehr wie dem unsern, wird Schneid und persönliches Draufgehn im Feuer verlangt werden und das allein legt den Gedanken nahe, vom Beginn des Erziehungswerkes eines Jahrgangs an die Jugend der Primaner sich mit der größeren Gesetztheit der Abiturienten paaren zu lassen. Wer sich in seinem Leben viel mit dem Offiziernachwuchs hat befassen müssen, die Eigenart der jungen Menschen auf sich hat einwirken lassen, der wird dem beitreten. Einer ergänzt den anderen und die Marine gewinnt nur dabei. Wenn mir schon dieserhalb eine nicht zu schwache Zahl von Primanern willkommen wäre, wie viel mehr noch, um mit ihrer Hilfe die uns unentbehrliche gesellschaftliche Mischung: Land, Armee, höherer Staatsbeamter neben den gut bürgerlichen Kreisen sicher zu stellen. Eine gesunde Mischung wird uns am besten vor der Einseitigkeit schützen, die eine Marine nicht brauchen kann. Von einer "Mischung « kann man aber nicht sprechen, solange der eine oder andere Bestandteil nur sporadisch, zufällig vertreten ist. Dieser verliert damit die Eigenschaft, im Korps heimisch zu sein. Unter Berücksichtigung der erreichbaren Möglichkeiten, wie die bisherige Erfahrung uns solche lehrt, halte ich also eine angemessene Zusammensetzung des Ersatzes aus nicht überalterten Abiturienten und begabten Primanern aus erwünschten Kreisen für die richtige.

Gründe, weshalb sidh die uns in größerer Zabl, als bisher, erwünschten Kreise zurückbalten.

An der Tatsache, daß statt der von uns bevorzugten Anwärter, Anmeldungen überwiegend aus Kreisen eingehn, die dem Offizierstande bisher ferner gestanden haben, kann nicht mehr gezweifelt werden ${ }^{125}$. Bis zu einem gewissen Grade geht es der Armee nicht besser. Es liegt das daran, daß sich das Zahlenverhältnis infolge der stark anwachsenden Bevölkerung insofern mehr und mehr zu unsern Ungunsten verschiebr, als jene Familien, denen wir unsere Offiziere gern entnehmen möchten, gegenüber andern Volksschichten an dem Wachstum nicht gleichen Anteil nehmen. Auch geht der Kinderreichtum an und für sich bei ihnen zurück; schließlich ist die Neigung dieser Kreise, die Söhne aus Existenzrüdksichten in gewinnbringende Berufsarten zu lenken ${ }^{126}$, heute stärker, als in einer verhältnismäßig wenig zurüdkliegenden Zeit. Auch ist man über die heutigen Ersatzverhältnisse in der Marine, auf dem Lande, in Offizier- und höheren Beamtenkreisen natürlich unterrichtet und empfindet häufig keine rechte Neigung mehr, den eigenen Sohn zu schicken. Interesse und Bewunderung für die Marine sind groß, meist jedoch platonisch: "der Sohn hat sich anders entschlossen ". In dem gleichen Maße, wie nun hier die Neigung, die Söhne zur Marine zu schidken abnimmt, wächst auch die Sicherheit auf der anderen Seite ${ }^{125}$. Da die Beispiele, wo man mit der Marine Glüdk hatte, immer zahlreicher werden, wird von der günstigen Gelegenheit, im Offizierkorps festen Fuß zu fassen, auch reichlicher Gebrauch gemacht. Je mehr aber von unten zufließt, desto mehr fließt von oben ab. Es ist das ein natürlicher, in unserer ganzen Gesellschaftsordnung begründeter Vorgang. Ich möchte in dieser Gedankenrichtung mit besonderem Nadhdruds auf die Gefahr hinweisen, daß die Verhältnisse, sofern es nicht noch glüdkt, den rollenden Stein aufzuhalten, sich rapide weiter zu unsern Unginsten verschieben müssen ${ }^{123}$. Der Seeoffizier wird vorwiegend in den Kreisen für die Marine

123 Krosigks Randbemerkung: "Mehr gute Primaner nothwendig.* "Also*.

124 Krosigks Randbemerkung: Auch meiner Oberzeugung nach. Die Abiturienten sollen das spritzige Salz bilden, eine Speise kann aber versalzen werden."

125 Der Satz ist am Rande durdh einen senkrechten Doppelstrich hervorgehoben.

120 Vgl. S. 4. Diese Entwicklung ist von Demeter, S. 27, eingehender behandelt worden. 
werben, in denen er selbst heimisch ist. Ein weiterer Grund des Rüdkganges ist der, daß die betreffenden Eltern erkannt haben, wie die Aussichten für Primaner in Anbetracht der grossen Zahlen der Abiturienten - worüber die Bestimmungen klaren Aufschluß geben - nur noch geringe sind. Die Bevorzugung der letzteren ist ihnen so deutlich vor Augen gerüdkt, daß sie keine Hoffnung mehr zu haben glauben, ihre Söhne bei uns in höhere Chargen gelangen zu sehn. Das lodkt aber den General ebenso wenig, wie den Herrn vom Lande. Sie wollen ihren Jungen nicht dauernd an der Queue marschieren und im Laufe einiger Jahre von vielen anderen übersprungen sehn. Der Sohn selbst war bei dem häufigen Ortswechsel des Vaters, den ungünstigen Schulverhältnissen bei Landangesessenen nicht in der Lage, das Reifezeugnis zu erwerben; oder aber - und auch das ist oft eine Folge der Eigenart des väterlichen Berufes - er war im Drange nach Taten und vermeintlich größerer Freiheit auf der Sdrule nicht länger zu halten. Man wird den Empfindungen der Eltern eine gewisse Berechtigung nicht absprechen können. In den Kreisen, um die es sich hier handelt, vornehmlich Militär- und Beamtentum, herrscht viel Tradition und Familienstolz; und damit stimmt es nicht überein, die Söhne einem Korps zu übergeben, in welchem sie zeitlebens und von vornherein vollkommen bewußt gegen andere zurüdsstehen sollen. Ihnen die Verhältnisse in milderer Form schildern zu wollen, kann man nach Lage der Dinge nicht mehr verantworten. Auch würde man in dem Punkte keinen Glauben finden ${ }^{127}$. Es ist in den letzten Jahren so viel Unzutreffendes und Obertriebenes über die Abiturientenfrage der Marine geschrieben ${ }^{128}$ und geredet worden, daß im Inlande die Ansicht, daß nur sie noch Aussicht hätten, feste Wurzeln gefaßt hat ${ }^{120}$. Vielfach herrscht die Ansicht, daß das Abiturium geradezu Bedingung sei. Wenn nun die Eltern, in der Erkenntnis, daß die Marine Primaner nicht haben will, ihre Söhne bis zum Abiturium in der Schule lassen, um erst dann bei uns einzutreten, ändern diese häufig ihre Sinnesart in den 2 Jahren, werden von wohlmeinender Seite der Marine entfremdet, kommen infolge von verdorbenen Augen oder aus anderen gesundheitlichen Gründen, für den Eintritt nicht mehr in Frage ${ }^{125}$. Die Eltern ziehn auch für ihre Söhne, wenn diese einmal die Schule absolviert haben, andere nach ihrer Ansicht sicherere oder größeren Gewinn versprechende Berufsarten häufig vor. Selten legen Eltern Wert darauf, ihre Söhne zur See gehen zu sehn, reden im günstigsten Fall weder ab noch zu. In dem jungen Manne selbst aber wächst mit zunehmendem Alter die Neigung, die kritische Sonde an die Dinge zu legen. Er hört dies und jenes, was ihm nicht gefällt, ihm sagen die Kreise nicht zu, denen die in seine Heimat beurlaubten Seeoffiziere und Fähnriche angehören, und die den Eltern bisher fremd waren. $\mathrm{Daß}$ diese häufig keine rechte Aufnahme in seinem heimatlichen Regiment finden, dem die eigene Familie traditionell verbunden ist, kann ihm nicht entgehen. Die Lebensansprüche und Auffassungen des jungen Mannes steigern sich in den oberen Schulklassen, lassen ihn viele Dinge mit anderen Augen schauen. Der Primaner beschäftigt sich mehr mit der Poesie, der Abiturient mit der Prosa, den realen Fragen des künftigen Berufs und - schwenkt ab. Ohne alle Frage verlieren wir gerade durch dieses Abschwenken vor Toresschluß eine außerordentliche große Zahl besten Menschenmaterials ${ }^{125}$. Der Einwurf hierzu, daß es kein Verlust sei, solche Leute von uns abwandern zu sehn, trifft in keiner Weise zu. Denn als Primaner wären dieselben jungen Leute noch mit Passion eingetreten und möglicher Weise vortreffliche Offiziere geworden. Die Eltern, bedächtiger als die Söbne, lassen sich, wie es scheint, auch häufig durch den zahlreichen Schund in der Marineliteratur einschüchtern, der es sich zur Aufgabe macht, dem Laien schaurige und falsche Schilderungen zu bringen, und eine ganz besondere Gefahr in politisch unsicheren Zeiten darstellt. Auch wäre es nütz-

127. Randbemerkung: ? a.

129 S. die entsprechenden Jahrgänge der Marine-Rundschau. Ferner: Seeoffizierlaufbahn, in: Berliner Correspondenz (18. 8. 1910); Seeoffizierlaufbahn und Abiturientenexamen, in: Hamburger Nachrichten (21. 1. 1912); Die Vorbildung für die Seeoffizierslaufbahn, in: Heer und Flotte (Juli 1912).

120 Krosigks Randbemerkung: «Bei rigoroser Durchführung der betreffenden Abiturienten-Vorschrift wird das doch auch der Fall sein.« 
licher, wenn nicht jeder Unfall bei uns in der Presse breitgetreten und ausgeschlachtet würde ${ }^{130}$. Die Laienkreise knüpfen allerhand falsche Kombinationen an derartigen Zeitungsklatsch und fraglos gehn auch hierdurch weitere junge Leute für uns verloren. Wenn man trotz allem bisher Erwähnten die Hoffnung auf irgend einen Ausweg vielleicht nicht aufgeben braucht, so ist ein anderer Punkt wohl wesentlich bedenklicher. Es scheint nämlich außer Zweifel, daß namentlich ein großer Teil des jungen Offizierkorps heute ohne rechte Begeisterung und Befriedigung im Binnenlande von dem erwählten Berufe spricht ${ }^{125}$. Trifft das in der Tat zu, so muß im Zusammenhange dieser Ausführungen festgestellt werden, daß dieser Umstand hauptsächlich wieder zu einer Einbuße an Anwärtern aus den hier erwähnten Kreisen führen müßte. Denn fraglos sind die Eltern vom Lande ängstlicher um die Zukunft ihres Sohnes besorgt, ihnen steigen mehr Bedenken, als den in großen Städten Lebenden auf. Die Söhne von höheren Beamten und Offizieren aber haben das Leben meist schon von einer etwas höheren Warte kennen gelernt. Aus ihrem ganzen bisherigen Milieu heraus haben sich ihre Erwartungen und Begriffe vom Leben wohl erklärlicher Weise gesteigert. Erfahren sie aus kritischen unzufriedenen Schilderungen, daß die Marine heute für den angestrengten und wenig unterbrochenen Dienst und viele Entbehrungen kein rechtes Aquivalent, keine Abwechselung, keine eigene Häuslichkeit, keinen Familienanschluß, kein Ausland bietet, so mag das wohl manchen unter ihnen zur Umkehr bringen.

Mir scheint, daß dieser Punkt unsere allergrößte Aufmerksamkeit erheischt 191. Durch Erlasse, Befehle, Unterweisungen ist da wenig oder nichts zu machen. Wohl aber scheint es ratsam, und ich habe es an besonderer Stelle des Berichts versucht, die Frage zu prüfen, ob wir etwa in der Lage wären, mit verfügbaren Mitteln, das Leben namentlich unserer jungen Offiziere befriedigender zu gestalten - selbstverständlich ohne dadurch einer etwaigen Verweichlichung oder Herabminderung der dienstlichen Leistungen Vorschub zu leisten. Idh möchte annehmen, daß sich in der Richtung etwas erreichen lassen wird.

Bestimmte Vorshläge für eine teilweise Revision der beutigen Eintrittsbestimmungen. 1.) Innerhalb der einzelnen Prädikate, vorzüglich, gut, binreichend 152, rangieren die Abiturienten vor den Primanern. [...] 193. Was sie [Primaner] heute erbittert, ist ihre Ohnmacht auch den notorisch schlechten, wenig taugenden Abiturienten gegenüber ${ }^{184}$. Sie treten nun einmal mit den Abiturienten gleichzeitig ein, tun den gleichen Dienst, erhalten denselben Unterricht; ihr Streben, sich mit ihnen zu messen, ist gut und menschlich, darf nicht gewaltsam niedergedrüdk werden. [...] ${ }^{136}$.

2.) Die Bestimmung bezüglich des Vorrechts der guten Abiturienten, mit der Beförderung zum Leutnant um ein Jahr im Besoldungsdienstalter vorzurüdken, sollte dahin ge-

1so „Geschieht leider durch die Marineangehörigen selbst. M.«

191 Krosigks Randbemerkung: "Urlaub ist das beste Mittel. M. E. sind sich darüber alle Kommandobehörden vollständig im klaren u. bedacht, den Offizieren p. p. jährlich ihre Urlaube zuzuwenden so weit es der drüdkende Personal-Mangel uns gestattet. Auf der Flotte war die jährliche Beurlaubung der Off. u. Mannschaften ganz schematisch geregelt.凶

192 Krosigks Randbemerkung, zu vorzügliक: »ja«; zu gut: »ja«; und zu binreidend: "nein*.

1ss Hier folgt eine Beschreibung, wie die Maßnahmen in der Praxis aussehen sollten: unter *vorzüglid, würden nur die Abiturienten in Anspruch genommen werden; unter "gut* kämen auch einige Primaner vor den nur binreichenden* Abiturienten.

194 Krosigks Randbernerkung: »ist sehr natürlich u. verständlid.. *Nur hinreichenden Abiturienten eine besondere Chanze zu bieten liegt m.E. nicht der geringste Grund vor, hier in der 3.ten Kategorie muß mindestens harte Konkurrenz stattfinden. Das bedeutet, 1.) einen Ansporn für Primaner sich einen besseren Platz zu erringen und damit die Möglichkeit verbesserter Aussichten und 2.) größeren Vortheil für die guten Abiturienten wenn sie vor Kategorie 3 der Vor-Crew rangirt werden.

195 Hier folgt der Gedanke, einigen *fixen und gewandten Primanern, auch wenn ihr theoretisches Wissen mit den vorwiegend praktischen Leistungen nicht Sohritt hält*, die Chance zu bieten, in Kategorie 2 mit den Abiturienten zu rangieren. 
ändert werden, daß dies, wie in der Armee, allen Abiturienten, nicht nur den guten, zustehn soll ${ }^{136}$. [...] ${ }^{137}$.

3.) Während in bezug auf die bevorzugte Gehaltsgewährung eine Erweiterung zu Gunsten der Abiturienten empfohlen wurde, scheint mir die durch die A.K.O. vom 2. Juli 1907 in Aussicht gestellte teilweise Vorpatentierung angesichts der ganzen Entwicklung unserer Personalverbältnisse seit Erlaß dieser Ordre nicht mehr erwünscht - auch ist mir eine andere Anschauung aus dem Offizierkorps heraus nicht bekannt geworden 198. Die über Erwarten gesteigerten Einstellungszahlen lassen sich ohne erhebliche Beteiligung von Primanern nicht mehr erreichen ${ }^{130}$. [...] ${ }^{140}$. Die Frage der Vorpatentierung wurde schon an anderer Stelle kurz erwähnt. Hier nur soviel, daß ich nicht glaube, daß die Primaner sich an diese Maßnahme gewöhnen werden ${ }^{141}$, nur weil es Bestimmung ist. Ein im Dienst bereits erprobter Offizier, der dies ruhig und unberührt hinnimmt, würde mir auch nicht gefallen. Man darf nicht übersehen, daß das Überspringen in der Armee im ersten Beginn der Offizierlaufbahn, bei uns dagegen zu einer Zeit erfolgen soll, wo die Betreffenden sich bereits in einigen Jahren des Dienstes gegenseitig gemessen haben, und daß die Ordre hierfür in einem Zeitpunkt engehn kann, wo der zu Überspringende als älterer auf demselben Schiffe mit dem Springenden Dienst tut. Das wird sich bei den großen in Frage kommenden Zahlen zumal im Auslande nicht vermeiden lassen. Berufsausbildung und Seeerfahrung sind bei der Beförderung zum Oberleutnant schon soweit fortgeschritten, daß die Unterschiede der Schulbildung sich zu verwischen beginnen. Nicht selten wird auf Grund der ersteren ein ehemaliger Primaner höher eingeschätzt als der ehemalige Abiturient; die gerechte Beurteilung als Grundlage für das Springen wird schwierig, die damit zu übernehmende Verantwortung ganz außerordentlich groß sein. Hinzu kommt, daß die Verbände der Jahrgänge, die sich gerade in letzter Zeit wieder zusammenfinden, durch diese Maßnahme auseinandergerissen werden. Die Bevorzugten werden aus ihnem Jahrgange entfernt, ohne aber in den nächst älteren kameradschaftlich aufgenommen und anerkannt zu werden. Besonders bedenklich, daß auch die besten, in den bisherigen Jahren der Praxis vielleicht glänzend bewährten Primaner übersprungen werden müssen. Läßt man aber nur einen Teil der Primaner überspringen, so wird dadurch ein Keil auch zwischen diese geschoben, dadurch erhöhte Kritik und Unzufriedenheit geschaffen. Vergegenwärtigt man sich den enormen Vorteil ${ }^{127}$ der heute für die Vorpatentierung in Frage kommenden Abiturienten, den diese an und für sich schon durch ihren Platz im eignen Jahrgang von mehreren hundert Köpfen haben, so stellt das allein doch eine außerordentliche Bevorzugung dar. Dem verhältnismäßig geringen Vorteil 142, die Primaner des nächsten Jahnganges zu überflügeln, aber stehen die angedeuteten großen und schwerwiegenden Nachteile auf moralischem Gebiete gegenüber. Beunruhigung und Unzufriedenheit wird in die weitesten Kreise getragen. Großer Schaden erwächst uns aus der vermeintlichen Erkenntnis bei den Eltern und ihren eintrittslustigen Söhnen, daß es in Zukunft eine erste und eine zweite Klasse von Seeoffizieren gibt ${ }^{145}$ und daß nur das Abiturium in ihren Augen - gleichgültig wie die Verhältnisse sonst liegen - zur ersten Klasse berechtigt. Ein Vergleid mit der Armee ist hierbei

130 Krosigks Randbemerkung: „Durch Besoldungsgesetz geregelt. M. E. Änderung nicht nötig. Allgemein wird es schon für alle am 3 . Jahr vorgerüdkt!*

197 Hier folgt die Argumentation, daß alle Abiturienten, nicht nur die mit der Bezeichnung "gut", auch im Besoldungsgesetz vorpatentiert werden sollten. Unter den jetzigen Bestimmungen bestehe »das unerwïnschte Odium*, daß »gute* Leistungen durch Geld anerkannt und daß Abiturienten, die nicht das Prädikat *gut * erhalten haben, moralisch gekränkt würden.

138 Krosigks Randbemerkung: "Ist überhaupt noch nicht in Erscheinung getreten, da erst Jahrgang 1909 davon berührt wird u. daher erst c. 1915/16 z. Ob.Lt.z.S. befördert wird.« Vgl. Anm. 52.

130 Vgl. S. 89, 92.

16 Hier folgt der Vorschlag, den Vorteil der Vorpatentierung für die Abiturienten in dem Maße zu verringern, »indem die Zahl der Primaner mit Hilfe der jetzigen Bestimmungen reduziert werden soll..

141 Krosigks Randbemerkung: "auch meiner Ansicht*.

142 Randbemerkung: " Ja!«

148 Randbemerkung: "Ubertreibung!^ 
nicht gut möglich, da die einzelnen Bevorzugten sich in den Riesenzahlen im Verhältnis zur Marine vollkommen verlieren. Meiner festen Uberzeugung nach ist die Vorpatentierung der springende Punkt, das "rote Tuch«, das viele erwünschte Elemente zurüdkschredkt. Nur von ihrer vollkommenen Beseitigung wird man eine wesentliche Besserung erwarten dürfen ${ }^{125}$. Es muß durch die Presse bekannt werden, daß aus Anlaß der unerwartet eingetretenen Personalverhältnisse infolge der Flottenvermehrung die Beibehaltung der Vorpatentierung auf zu große Schwierigkeiten stößt. Eine verständliche Erklärung hierfür zu geben, kann nicht schwer halten ${ }^{127}$. In den unter den heutigen Bestimmungen eingetretenen Jahrgängen würden immerhin eine angemessene Zahl von Vorpatentierungen seiner Zeit vorzunehmen sein.

4.) Sehr notwendig ist eine ruhige, sachliche Aufklärung in der guten Presse; mit dem Ziel, daß der Abiturient uns nach wie vor besonders schätzenswert ist, wenn er auch den sonstigen Forderungen gerecht wird, daß wir daneben aber auf den nicht zu alten geistig und körperlich gur beanlagten Primaner keineswegs verzichten wollen. [...] ${ }^{144 .}$ Zum Schluß möchte ich meinen unmaßgeblichen Eindruck dahin zusammenfassen, daß wir uns, wie die Dinge heute liegen, mehr und mehr dem unerwünschten Ziele nähern: Mittelmäßige Abiturienten (bezogen auf die Gesamtsumme der zu fordernden Eigenschaften) und soblechte Primaner ${ }^{125}$. Kleine Mittel werden nicht mehr helfen. Es muß schnell und energish durchgegriffen werden. Schnell auch mit Rüdssicht darauf, daß der Bedarf der Armee wohl schon im kommenden Jahre eine erhebliche Steigerung erfahren wird, was uns recht unbequeme Rückschläge bringen könnte. Gegen die eine teilweise Anderung der Bestimmungen erstrebenden Anregungen könnte nun eingewendet werden, daß diese noch nicht genügend lange in Kraft seien, um in ihrer endgültigen Wirkung beurteilt werden zu können. Das träfe jedoch keinesfalls zu. Denn an der Tatsache wird nicht mehr gezweifelt, daß wir uns in sozialer Richtung in einer Abwärtsbewegung befinden ${ }^{125}$. Ferner aber würde es sich, nachdem diese Tendenz nach unten klar erkannt ist, angesichts der heutigen Einstellungszahlen um ein Experiment handeln, dessen Folgen sich in einem Menschenalter nicht wieder verwischen lassen. Mit jedem Jahr wächst der Schaden und zwar in potenzierter Form. Wir sind bekanntlich nicht die einzige Marine, die gerade in dieser wichtigsten aller Fragen Enttäuschung erlebt. Wir wissen aber auch, daß andere Marinen die Dinge zulange haben anstehn lassen, so daß der rechte Moment zur Umkehr verpaßt wurde. Schließlich möchte ich noch der etwaigen Befürchtung entgegentreten, daß wir eine zu starke Einbuße an Abiturienten durch Anderung der heutigen Ejntrittsbestimmungen erfahren müßten. Hiergegen schützt uns der allgemeine Zug unserer Zeir, den Söhnen eine abgeschlossene Schulbildung zu geben und zwar das aus rein eigennütziger Überlegung, die im Grunde keinerlei Anspruch auf Entschädigung verdient oder auch nur beansprucht.

v. Dambrowski 
